\title{
Pricing for Delivery Time Flexibility
}

\author{
Baris Yildiz ${ }^{1}$ and Martin Savelsbergh ${ }^{2}$ \\ ${ }^{1}$ Department of Industrial Engineering, Koc University, Istanbul, Turkey \\ ${ }^{2}$ H. Milton Stewart School of Industrial \& Systems Engineering, Georgia Institute of \\ Technology, Atlanta, USA
}

April 18, 2019

\begin{abstract}
We study a variant of the multi-period vehicle routing problem, in which a service provider offers a discount to customer in exchange for delivery flexibility. We establish theoretical properties and empirical insights regarding the intricate and complex relation between the benefit from additional delivery flexibility, the discounts offered to customers to gain additional delivery flexibility, and the likelihood of acceptance of discount offers by customers. Computational experiments, using an exact dynamic programming algorithm show that, depending on the setting, cost savings exceeding $30 \%$ can be achieved.
\end{abstract}

keywords: multi-period routing, flexibility, pricing, discount, acceptance probability

\section{Introduction}

Increasing the flexibility to schedule deliveries can be advantageous, because it may allow consolidations that were previously impossible. For example, if it is possible to adjust the time windows during which deliveries can be made at two customers that are geographically close, it may be possible to schedule these deliveries on the same route, whereas the original time windows may have prevented this. Or, if it is possible to adjust the delivery day for two customers that are geographically close, it may be possible to schedule these deliveries on the same day, whereas the original delivery day requirements may have prevented this. Thus, increasing the flexibility to schedule deliveries can result in reduced delivery costs. Consequently, it is natural to consider offering discounts to customers in return for increased delivery flexibility. The discount offered should, of course, depend on the benefit, i.e., the cost reduction, that will result from the additional flexibility, and on the likelihood of the discount offer being accepted.

To study the intricate and complex relation between the benefit from additional delivery flexibility, the discount offered to gain additional delivery flexibility, and the likelihood of acceptance of discount offers, we study a variant of the multi-period vehicle routing problem, in which a service provider offers a discount in exchange for delivery flexibility. More specifically, we consider a setting in which customers place orders to be delivered during a planning horizon consisting of several consecutive days. Each customer has an associated revenue, delivery quantity, delivery location, and preferred delivery day. The service provider offers a discount to its customers in exchange for 
flexibility in the timing of the delivery, for example, the delivery is allowed to be made one day early or one day late (one day before or one day after the preferred day). Estrada-Moreno et al. (2019) analyze the potential benefits for a given, pre-specified discount. Here, we consider go further, and simultaneously determine the optimal discount offers and the optimal delivery routing taking into account the fact that customers may reject a discount offer.

To simplify the analysis and interpretation of the results, we restrict ourselves to a single-vehicle problem and delivery locations on a line, which implies that the daily routing problem is easy. It is intuitively clear that offering discounts to customers one by one, i.e., a next discount offer is determined after the outcomes of previous discounts offers have been observed, even though this may be impractical, should result in greater benefits than offering discounts simultaneously to all customers. We formally establish this property. We also characterize the situations in which there is no benefit to be gained from offering discounts to customer one by one. Furthermore, we analyze the performance of natural offer sequences, e.g., offer discounts to customers in non-decreasing order of their distance to the depot, under different assumptions on the discount offer acceptance probability functions.

To be able to gain empirical insights, in addition to some theoretical properties, we develop a dynamic programming algorithm for solving small to medium size instance to optimality. An extensive computational study shows the potential benefits of offering discounts to gain delivery flexibility, which, depending on the specific setting, can exceed $30 \%$.

The remainder of the paper is organized as follows. In Section 2, we discuss relevant literature. In Section 3, we formally introduce the Pricing for Delivery Flexibility Problem (PDFP), establish a number of theoretical properties of the PDFP, and present a dynamic programming algorithm for the exact solution of instances of the PDFP. In Section 4, we discuss the results of an extensive computational study. We conclude, in Section 5, with some final remarks.

\section{Related literature}

The PDFP belongs to the class of multi-period vehicle routing problems (MPVRP), which was first studied in the context of waste collection (Beltrami and Bodin, 1974). For a comprehensive coverage of the MPVRP and its variants, we refer the reader to Mourgaya and Vanderbeck (2006) and Francis et al. (2008).

Below, we focus on studies that involve problem settings similar to the PDFP, that perform a similar type of analysis, or that employ a similar solution approach.

The PDFP considers demand management in a logistic environment, a research area which has started to receive more and more attention in the academic community, e.g., Agatz et al. (2011); Coelho et al. (2012); Yang et al. (2014); Yildiz and Savelsbergh (2019a). The goal of demand management is to increase profit. However, whereas in traditional demand management studies, the focus has been on increasing revenue (see, for example, Talluri and van Ryzin (2004)), as the cost is fixed, e.g., airline flight, hotel room, in the context of logistics, the cost is not (necessarily) fixed and demand management can also be used to reduced costs, which is the focus of our research.

Demand management in logistics can be passive, i.e., exploiting existing flexibility, or active, creating additional flexibility by offering incentives. The significant potential of exploiting service time flexibility to reduce delivery costs in MPVRP applications, e.g., passive demand management, has been investigated in several studies, e.g., (Wang and Toktay, 2008; Archetti et al., 2015b, 2017). Archetti et al. (2015b) investigate a variant, in which customers have to be served between 
a release and a due date and in which customers with due dates exceeding the planning horizon can be postponed at a cost. The objective is to find vehicle routes for each period such that the total costs, i.e., transportation costs, inventory holding costs, and postponement costs, is minimized. The authors propose alternative mixed integer programming (MIP) formulations and present a branchand-cut algorithm for their solution. In a recent study, Estrada-Moreno et al. (2019) extend the work of Archetti et al. (2015b) by introducing the possibility to offer price discounts to gain service time flexibility. The authors propose a local search heuristic to find low-cost solution, which includes both the transportation costs and the cost of the price discounts offered. Results of an extensive computational study show the potential of pricing for delivery flexibility. However, contrary to our research, the authors assume that the price discounts are given and that the customers will always accept a discount offer.

Angelelli et al. (2007a,b) consider a setting in which service providers have the flexibility to serve customers on the day after an order is placed, or on the following day. When planning deliveries for today, all information about orders is known (a set of orders that has to be delivered as these orders were postponed yesterday and the set of orders placed yesterday), but information about tomorrow is uncertain (the set of orders that will be postponed today and the set of orders that will be placed today). Ulmer et al. (2018) consider a variant in which orders placed today can be rejected, or can be served today or tomorrow. Acceptance of orders, postponement of orders, and routing decisions therefore have to anticipate additional orders today and tomorrow. The authors model the problem as a dynamic vehicle routing problem with stochastic service requests and propose an approximate dynamic programming approach for its solution.

The distinguishing feature of our research is that it focuses on active demand management, i.e. offering discounts in return for additional service time flexibility. To the best of our knowledge, $\mathrm{Li}$ et al. (2012) were the first to investigate the value of adjusting prices (offering discounts) to increase delivery flexibility and, thus, to decrease logistics cost. The authors explore that idea in the context of the single-item, uncapacitated lot-sizing problem, and the results of a comprehensive computational study demonstrate that significant cost reductions can be achieved.

In this paper, we focus on instances of the PDFP with customer locations on a line segment and the depot at one of its end points so as to simplify the exposition and to be able to derive analytical results and insights that are independent of the routing decisions. Studies involving simple network topologies, i.e., half lines or star networks, which eliminate the need for complex vehicle routing decisions, are frequently used to study aspects of delivery problems, e.g., Angelelli et al. (2007a,b); Archetti et al. (2015a); Klapp et al. (2016); Reyes et al. (2018); Yildiz and Savelsbergh (2019b). In fact, this not only happens in the study of delivery problems, but also in other applications, e.g., Zhang et al. (2002); Emde and Boysen (2014); Erdoğan et al. (2014).

We develop a dynamic programming algorithm for the solution of these instances of the PDFP. We show that the PDFP is challenging even for these instances as the state space already becomes prohibitively large for moderate-size instances. To address this issue, we propose the use of specific offer sequence that enables a drastic reduction in the size of the state space that needs to be explored by the dynamic programming algorithm. We refer reader to Morin (1978) and Bertsekas (2005) for comprehensive reviews of techniques for handling large state spaces in dynamic programming algorithms. 


\section{Model}

In this section, we present the basic setting we consider as well as the basic approach we use for modeling and solving the flexible delivery planning problem. Later we will discuss various extensions to this basic setting and their theoretical and practical implications.

There is a single depot located at one end of the line segment $[0,1]$, which represents the service region. There is a single vehicle with capacity $Q$ to deliver orders to customers. We assume a planning horizon of multiple days, i.e., $t=1, \ldots, T$. On any given day, the vehicle will perform a single delivery tour. Each order $i$, for $i \in I$, has an associated weight $q_{i} \leq Q$, preferred delivery day $t_{i} \in\{1, \ldots, T\}$, a distance to the depot $d_{i} \in(0,1]$, and revenue $r_{i}$. We assume that the total weight of delivery/pickup orders with preferred day $t$, for $t=1, \ldots, T$, is less than or equal to the vehicle capacity, i.e., we have $\sum_{i \in I: t_{i}=t} q_{i} \leq Q$, so that a feasible delivery plan exists. For each order $i$, the probability of accepting a delivery on day $t$ given a discount offer $\alpha$ is denoted by $P_{i}(\alpha, t)$. If the discount offer is not accepted, then $i$ has to be delivered on its preferred delivery day $t_{i}$. Thus, we have $P_{i}\left(\alpha, t_{i}\right)=1$ for any $\alpha$.

A delivery plan states which orders are to be served on which day. A delivery plan is capacity feasible (feasible in short) if on each day, both the total weight of the customers served is less than the vehicle capacity. The cost of a delivery plan is defined as the total distance traveled by the vehicle to perform the planned delivery routes.

A discount offer $(\alpha, t)$ to customer $i$, specifies the discount fraction $\alpha \in[0,1]$ offered to serve the order on day $t$. The expected cost of offer $(\alpha, t)$ to customer $i$ is defined to be the expected loss of revenue, i.e., $P_{i}(\alpha, t) \alpha r_{i}$. We call an offer $(\alpha, t)$ to customer $i$ empty, if $\alpha=0$ and $t=t_{i}$. We assume the delivery company knows all orders before it offers discounts to persuade acceptance of an alternative delivery day. (Note that the company makes a single offer to a customer for a particular day.) An offer policy specifies the sequence in which discount offers are made to the customers as well as the discount offers to be made for any possible sequence of offer outcomes. The Pricing for Delivery Flexibility Problem (PDFP) is to find an optimal offer policy that minimizes the total expected cost, i.e., delivery costs and discount costs.

\subsection{Dynamic programming algorithm (DP)}

We suggest to solve PDFP by using a dynamic programming algorithm that explores a finite number of states. We define a state $\sigma=\left\{L_{1}^{\sigma}, \ldots, L_{T}^{\sigma}\right\}$ as a collection of lists, one for each day $t \in\{1, \ldots, T\}$. The list $L_{t}^{\sigma}$, for $t=1, \ldots, T$, specifies the set of orders to be served on day $t$. The total weight of the orders in a list $L$ is denoted by $q(L)$. For a non-empty list $L$, the distance to furthest customer in the list is denoted by $\delta(L)$. We say that a state is empty if $L_{t}^{\sigma}=\emptyset$ for $t=1, \ldots, T$ and complete if $\bigcup_{t=1, \ldots, T} L_{t}^{\sigma}=I$; a state that is neither empty nor complete is called a partial state. The set customers contained in a state $\sigma$ (i.e., contained in one of its lists) is denoted by $I(\sigma)$. We define $n(\sigma)$ to be $|I(\sigma)|+1$, i.e., the empty state $\sigma^{\emptyset}$ has $n\left(\sigma^{\emptyset}\right)=1$ and a complete state $\sigma$ has $n(\sigma)=|I|+1$.

An offer policy $\mathcal{O}$ specifies an offer sequence $\pi^{\mathcal{O}}$ and a discount offer $o^{\mathcal{O}}(\sigma)$ for every possible state $\sigma$. We denote the customer receiving an offer in state $\sigma$ under policy $\mathcal{O}$ by $i^{\mathcal{O}}(\sigma)$. Note that $i^{\mathcal{O}}(\sigma)=\pi_{n(\sigma)}^{\mathcal{O}}$. When the policy is clear from the context we omit the superscript $\mathcal{O}$ and simply use $\pi, o(\sigma)$, and $i(\sigma)$.

An expansion $E(\sigma)$ of state $\sigma$ is obtained by inserting $i(\sigma)$ in lists $L_{t}^{\sigma}$ for $t=1, \ldots, T$. The set of states associated with order sequence $\pi$ is denoted by $\mathcal{S}(\pi)$. The leaf states $\mathcal{L}(\pi) \subset \mathcal{S}(\pi)$, for 
some offer sequence $\pi$, are the complete states representing delivery plans. The value $V(\sigma)$ of a leaf state $\sigma$ is set to $\infty$ if the state is infeasible, i.e., if $q\left(L_{t}^{\sigma}\right)>Q$ for at least one $t \in\{1, \ldots, T\}$, otherwise it is set to the total cost of the delivery plan, i.e., $\sum_{t=1}^{T} 2 \delta\left(L_{t}^{\sigma}\right)$. The value of a partial state $\sigma$ is defined as:

$$
V(\sigma)=\min _{t \in\{1, \ldots, T\}}\left\{\hat{V}\left(\sigma_{t}\right): \sigma_{t} \in E(\sigma, i(\sigma))\right\}
$$

where

$$
\hat{V}\left(\sigma_{t}\right)=\min _{\alpha \in[0,1]} P_{i(\sigma)}(\alpha, t)\left(\alpha r_{i(\sigma)}+V\left(\sigma_{t}\right)\right)+\left(1-P_{i(\sigma)}(\alpha, t)\right) V\left(\sigma_{t_{i(\sigma)}}\right)
$$

Let $t^{*} \in\{1, \ldots, T\}$ be the day for which we have $V(\sigma)=\hat{V}\left(\sigma_{t^{*}}\right)$, the chosen day of an interior state $\sigma$ and let $\alpha^{*}$ be the discount that minimizes the expected cost of the chosen day (i.e., solves (2) for $\left.V\left(\sigma_{t^{*}}\right)\right)$, the chosen discount of the chosen day for state $\sigma$. We call an offer $o^{*}(\sigma)=\left(\alpha^{*}, t^{*}\right)$ the optimal offer for state $\sigma$.

The value $V\left(\sigma^{\emptyset}\right)$ at the root of the decision tree gives the minimum expected total delivery and discount costs of the optimal offer policy for a given offer sequence $\pi$. At any interior state $\sigma$, the optimal offer is determined by the chosen day and the chosen discount. Thus, the dynamic program produces optimal offers for a given offer sequence $\pi$ and an associated minimum expected cost. The pseudo code for DP is presented in Algorithm 1, which uses the subroutine getValue (see Algorithm 2) to recursively calculate the optimal offer policy.

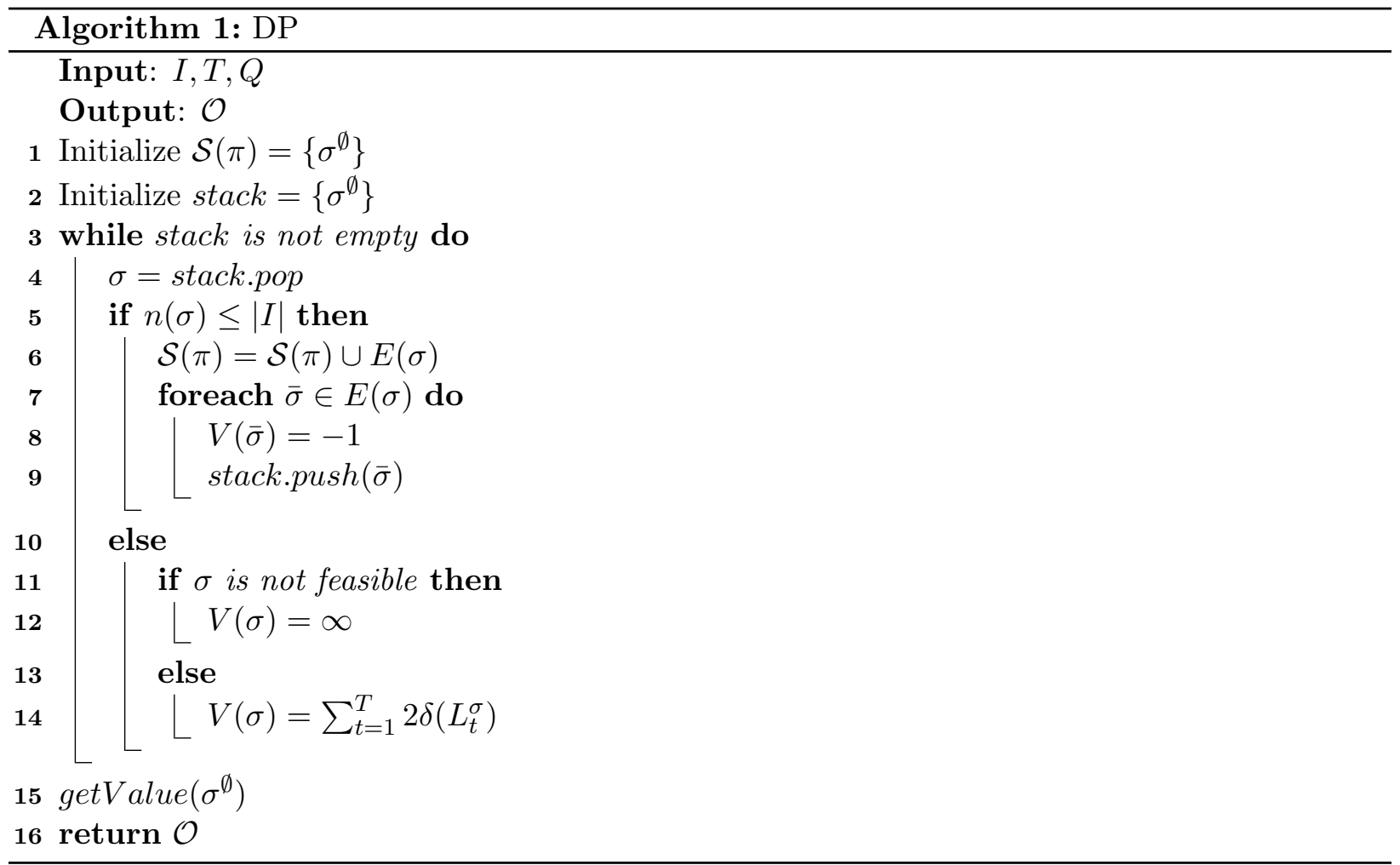

Next, we explore whether different offer sequences can result in different minimum expected costs, and, if so, whether there are classes of instances for which the offer sequence does not matter. 


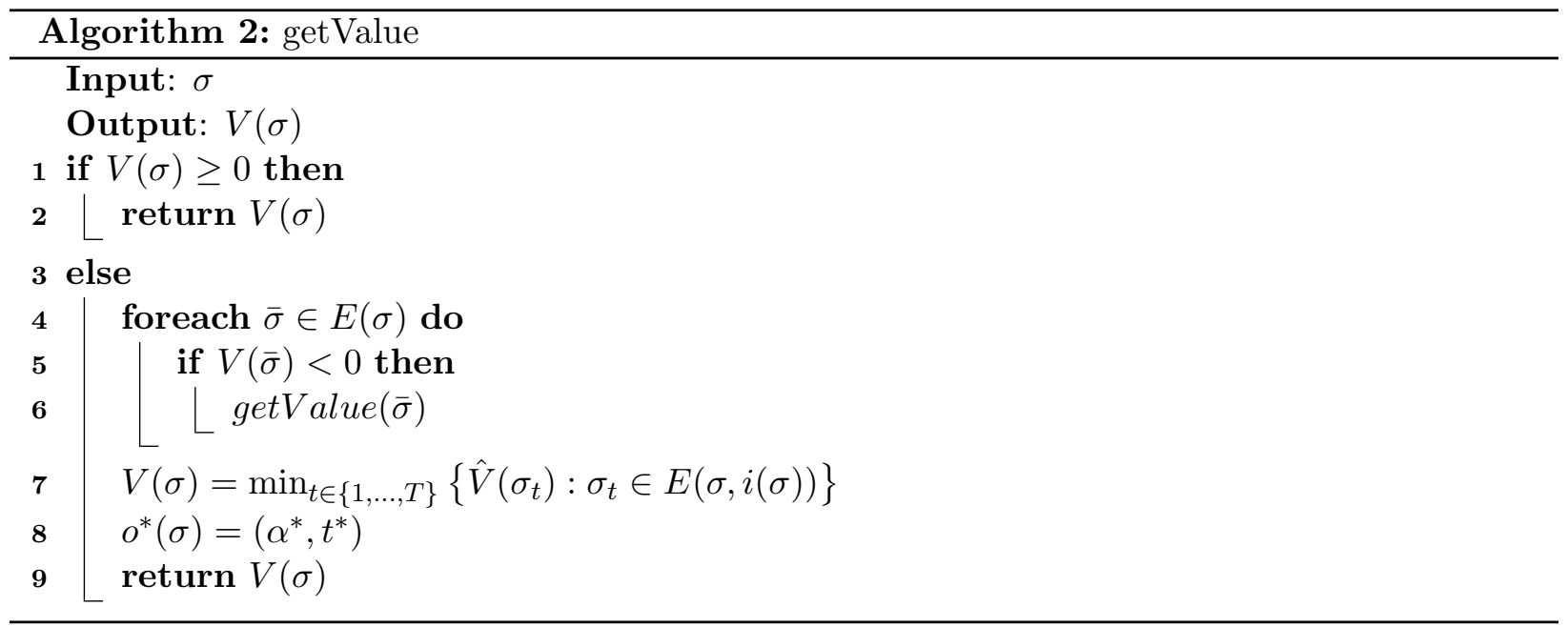

We start by presenting a small example, with two demands, two days, and a vehicle with capacity $Q=10$, which shows that different offer sequences can result in different minimum expected costs. The customer characteristics are shown in Table 1.

Table 1: Customer characteristics of the small example.

\begin{tabular}{r|r|r}
\hline$i$ & 1 & 2 \\
\hline$r_{i}$ & 10 & 10 \\
$d_{i}$ & 0.5 & 1 \\
$t_{i}$ & 1 & 2 \\
$q_{i}$ & 5 & 5 \\
$P_{i}(\alpha, 1)$ & $\min \{\alpha, 1\}$ & 1 \\
$P_{i}(\alpha, 2)$ & 1 & $\min \{10 \alpha, 1\}$ \\
\hline
\end{tabular}

The optimal offers for offer sequence $(1,2)$ and $(2,1)$ are shown in Figures 1a and 1b, respectively, which reveals that the minimum expected cost for offer sequence $(1,2)$ less than the minimum expected cost for offer sequence $(2,1)$.

Next, we define a number of concepts that help further explore the impact of different offer sequences on the minimum expected cost.

Definition 1. A PDFP instance has the sequence-independence property if for all possible offer sequences, the optimal discount offers result in the same minimum expected cost.

Definition 2. A PDFP instance has the sequence-free property if making optimal simultaneous discount offers (i.e., making discount offers to the customers simultaneously, thus foregoing the opportunity to decide discount offers based on the outcomes of previous discount offers) results in the same minimum expected cost as the optimal discount offers for the optimal offer sequence.

Intuitively, it is clear that the flexibility to decide a discount offer based on the outcomes of previous discount offers, as opposed to having to make all discount offers simultaneously, may have advantages for some instances. The following lemma formally establishes this result. 


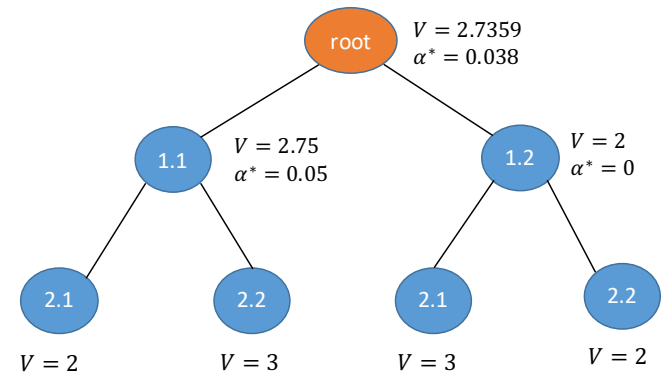

(a) $\pi=(1,2)$

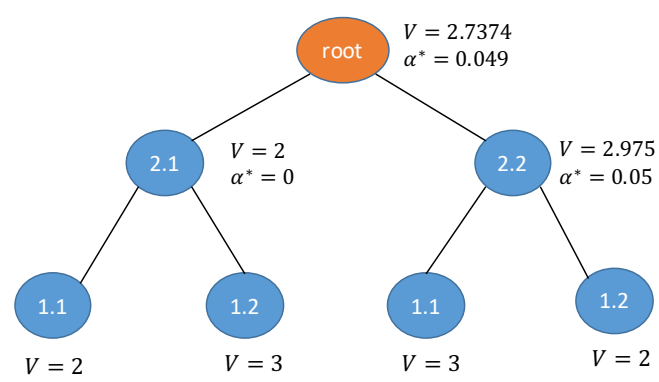

(b) $\pi=(2,1)$

Figure 1: The effect of offer sequence on minimum expected cost

Lemma 1. If, for a given PDFP instance, making optimal simultaneous discount offers results in the minimum expected cost, then the optimal discount offers for any offer sequence give the same minimum expected cost.

Proof. Let $o^{*}$ be the optimal set of discount offers when making simultaneous offers, let $o_{i}^{*}$ denote the offer made to customer $i$, let $P\left(\sigma \mid o^{*}\right)$ be the probability of ending up with the delivery plan represented by a leaf state $\sigma \in \mathcal{L}$, and let $\mathbb{E}\left(o^{*}\right)$ be the expected cost of the set of discount offers $o^{*}$. Because we have that for a given set of simultaneous offers $o$, the probability $P(\sigma \mid o)$ for $\sigma \in \mathcal{L}$ can be calculated easily, we have that the expected cost $C^{*}$ when making simultaneous discount offers $o^{*}$ is given by

$$
C^{*}=\sum_{\sigma \in \mathcal{L}}\left[P\left(\sigma \mid o^{*}\right) V(\sigma)\right]+\mathbb{E}\left(o^{*}\right)
$$

where the first term represents the expected cost of the delivery plan and the second term represents the expected cost of the discount offers.

Now, let $\pi$ be an arbitrary offer sequence and define the set of associated discount offers $\hat{o}$ as follows: for each state $\sigma$ in $\mathcal{S}(\pi)$, set $\hat{o}_{\sigma}=o_{i(\sigma)}^{*}$. Because we have that for a given set of (sequential) offers $o$, the probability $P(\sigma \mid o)$ for $\sigma \in \mathcal{L}(\pi)=\mathcal{L}$ can also be calculated easily, we have that the expected cost $V_{\pi}\left(\sigma^{\emptyset}\right)$ for the offer sequence $\pi$ and the discount offer set $\hat{o}$ is given by

$$
V_{\pi}\left(\sigma^{\emptyset}\right)=\sum_{\sigma \in \mathcal{L}(\pi)}[P(\sigma \mid \hat{o}) V(\sigma)]+\mathbb{E}(\hat{o})
$$

Because we have $P(\sigma \mid \hat{o})=P\left(\sigma \mid o^{*}\right)$ for $\sigma \in \mathcal{L}(\pi)$ and $\mathbb{E}(\hat{o})=\mathbb{E}\left(o^{*}\right)$, we have $V_{\pi}\left(\sigma^{\emptyset}\right)=C^{*}$.

Lemma 1 shows that PDFP instances that have the sequence-free property, also have the sequence-independence property. However, as we show next, the reverse is not true.

Proposition 1. There exist PDFP instances with the sequence-independence property that do not have the sequence-free property.

Proof. Consider the following example with two customers, two days, $t_{1}=1$ and $t_{2}=2$. Let $V_{A}$ be the delivery cost of serving the two orders on the same day and $V_{B}$ be the delivery cost for serving the two orders on different days. Assume that $P_{1}(\alpha, 2)=P_{2}(\alpha, 1)$ for $\alpha \geq 0$, i.e., the acceptance 
probabilities are identical (because we also have $P_{1}(\alpha, 1)=P_{2}(\alpha, 2)=1$ for $\alpha \geq 0$ ). Clearly, offer sequences $\hat{\pi}=(1,2)$ and $\bar{\pi}=(2,1)$ will give the same minimum expected cost. Let $\alpha_{1}^{*}$ and $\alpha_{2}^{*}$ be the optimal offers to Customer 1 and 2, respectively, when making simultaneous discount offers. For notational simplicity, we define $p_{1}=P_{1}\left(\alpha_{1}^{*}, 2\right)$ and $p_{2}=P_{2}\left(\alpha_{2}^{*}, 1\right)$. Then the minimum expected cost of the simultaneous offer policy, $C^{*}$, is

$$
C^{*}=V_{A}\left(p_{1}\left(1-p_{2}\right)+\left(1-p_{1}\right) p_{2}\right)+V_{B}\left(p_{1} p_{2}+\left(1-p_{1}\right)\left(1-p_{2}\right)\right) .
$$

Now consider the sequence $\hat{\pi}$ and its associated state space $\mathcal{S}(\hat{\pi})$ as illustrated in Figure 2 . In

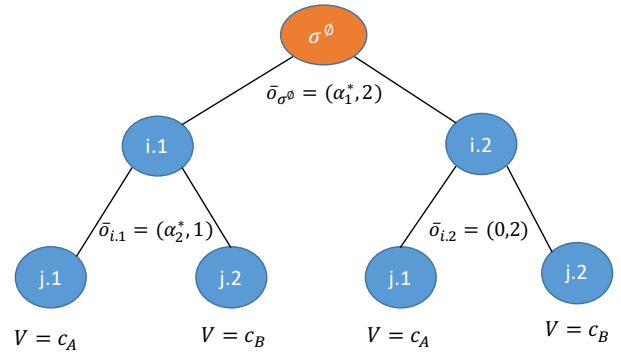

Figure 2: State space $\mathcal{S}(\hat{\pi})$

the figure, we also indicate the discount offers, i.e., $\left(\alpha_{1}^{*}, 2\right)$ to Customer 1 , and $\left(\alpha_{2}^{*}, 1\right)$ to Customer 2 if Customer 1 does not accept the offer and $(0,2)$ to Customer 2 if Customer 1 does accept the offer. The resulting expected cost (for these discount offers) is

$$
V_{\hat{\pi}}\left(\sigma^{\emptyset}\right)=V_{A}\left(\left(1-p_{1}\right) p_{2}+p_{1}\right)+V_{B}\left(1-p_{1}\right)\left(1-p_{2}\right) .
$$

But then we have $C^{*}-V\left(\sigma^{\emptyset}\right)=\left(V_{B}-V_{A}\right) p_{1} p_{2}$, which is strictly positive when $V_{B}>V_{A}$ and $p_{1} p_{2}>0$.

The proof of Proposition 1 provides insight into which PDFP instances may have the sequencefree property. Building on this insight, we next show that for PDFP instances in which the benefits of demand management (i.e., delivery cost reductions) are significantly larger than the costs of demand management (i.e., loss of revenue due to discount offers), making simultaneous discount offers can be optimal (i.e., result in the minimum expected cost).

Let $o_{\alpha}(\sigma)$ denote the value of the discount and $o_{t}(\sigma)$ the day of the offer, respectively, of offer $o(\sigma)$.

Proposition 2. Let $\pi^{*}$ be an optimal offer sequence and let $o^{*}(\sigma)$, for $\sigma \in \mathcal{S}\left(\pi^{*}\right)$, be the associated optimal discount offers for a given PDFP instance. If $P_{i(\sigma)}\left(o_{\alpha}^{*}(\sigma), o_{t}^{*}(\sigma)\right) \in\{0,1\}$, for all $\sigma \in \mathcal{S}\left(\pi^{*}\right)$, then the PDFP instance has the sequence-free property.

Proof. If $P_{i(\sigma)}\left(o_{\alpha}^{*}(\sigma), o_{t}^{*}(\sigma)\right) \in\{0,1\}$, for all $\sigma \in \mathcal{S}\left(\pi^{*}\right)$, then there exists a unique leaf state $\sigma^{*}$, for which we have $P\left(\sigma^{*} \mid o^{*}\right)=1$ and the minimum expected cost is given by

$$
V_{\pi^{*}}\left(\sigma^{\emptyset}\right)=V\left(\sigma^{*}\right)+\mathbb{E}\left(o^{*}\right) .
$$


Now consider the unique path from the $\sigma^{\emptyset}$ to $\sigma^{*}$ and let $\hat{o}$ represent the set of offers received by the customers on this path. Observe that when making this set of offers simultaneously, the probability of state $\sigma^{*}$ is one. Thus, the expected $\operatorname{cost} C_{\hat{o}}$ when making simultaneous discount offers $\hat{o}$ is

$$
C_{\hat{o}}=V\left(\sigma^{*}\right)+\mathbb{E}(\bar{o})
$$

The result follows from the fact that $\mathbb{E}(\hat{o})=\mathbb{E}\left(o^{*}\right)$.

In various settings threshold probability functions can accurately represent customer behaviors (i.e., accepting the offer if its higher than some threshold level specific to the customer). The following corollary to Proposition 2 shows that in such cases PDFP has the simultaneity property which then implies that it has anonymity property as well.

Corollary 1. If, for a given PDFP instance, the offer acceptance probabilities are step functions, i.e.,

$$
P_{i}(\alpha, t)=\left\{\begin{array}{ll}
1 & \text { if } \alpha \geq \alpha_{i}^{t}, \\
0 & \text { o.w. }
\end{array} \quad \forall i \in I, t \in\{1, \ldots, T\}\right.
$$

where $\alpha_{i}^{t}$ is the minimum discount demanded by a customer $i \in I$ to accept delivery on a day $t \in\{1, \ldots, T\}$, then the PDFP instance has the sequence-free property.

Proposition 2 and Corollary 1 have important implications, both from a practical and a computational efficiency perspective. Threshold acceptance probabilities (and more broadly acceptance probabilities satisfying Proposition 2) are likely to occur in real-life situations, and the fact that in that case simultaneously making discount offers results in the minimum expected cost, significantly simplifies implementation. From a computational efficiency perspective, as we will discuss in more detail in the following sections, Proposition 2 can be exploited to achieve drastic performance improvements.

For settings where the conditions of Proposition 2 do not hold, it is natural to ask whether it is possible to characterize effective, although not necessarily optimal, offer sequences. In the remaining of this section, we seek to answer this question, at least partially.

There are two characteristics that distinguish customers. First, the likelihood that they accept a discount offer to change their delivery day. Second, the impact of their decision on the delivery costs. Next, we explore these aspects in more detail.

Consider an instance with two customers, two days, identical revenues, order sizes that allow the orders to be delivered together on a single day, different preferred delivery days, and different distances to the depot. Such an instance has the property that the customers' decisions have the same impact on the cost of the delivery plan. If one customer accepts the offer to change its delivery day, then the customers are visited together on the same day, and no deliveries are made on the other day. If neither customer accepts the offer to change its delivery day, then each will be visited on one of the days. In what follows, we present analytical and numerical analyses that suggest that in this setting, it is best to first make an offer to the customer that is less likely to accept an offer to change its delivery.

We start by characterizing the optimal discount to offer to a customer for a specific family of offer acceptance functions. 
Lemma 2. Consider a PDFP instance with two delivery days and a state $\sigma$ with two child states $\sigma_{t_{i(\sigma)}}$ and $\sigma_{\bar{t}_{i(\sigma)}}$. If customer $i(\sigma)$ has an acceptance probability function of the form $p_{i(\sigma)}\left(\alpha, \bar{t}_{i(\sigma)}\right)=$ $\min \left\{\beta \alpha^{k}, 1\right\}$, then the optimal offer $\alpha^{*}$ is

$$
\alpha^{*}= \begin{cases}0 & \text { if } V\left(\sigma_{t_{i(\sigma)}}\right) \leq V\left(\sigma_{\bar{t}_{i(\sigma)}}\right) \\ \min \left\{\bar{\alpha}, \frac{k\left(V\left(\sigma_{t_{i(\sigma)}}\right)-V\left(\sigma_{\bar{t}_{i(\sigma)}}\right)\right)}{(k+1) r_{i(\sigma)}}\right\} & \text { o.w. }\end{cases}
$$

where $\bar{\alpha}=\left(\frac{1}{\beta}\right)^{\frac{1}{k}}$, is the minimum discount that guarantees acceptance of the offer.

Proof. Clearly, $V\left(\sigma_{t_{i(\sigma)}}\right) \leq V\left(\sigma_{\bar{t}_{i(\sigma)}}\right)$ implies that it is more cost efficient (in state $\sigma$ ) to serve customer $i(\sigma)$ on its preferred day, so $\alpha^{*}=0$.

If $V\left(\sigma_{t_{i(\sigma)}}\right)>V\left(\sigma_{\bar{t}_{i(\sigma)}}\right)$, we can rewrite (2) as follows, by substituting the acceptance probability function under consideration, i.e.,

$$
\alpha^{*}=\operatorname{argmin}_{\alpha \in[0,1]} f(\alpha),
$$

where $f(\alpha)=V\left(\sigma_{t_{i(\sigma)}}\right)\left(1-\beta \alpha^{k}\right)+\left(V\left(\sigma_{\bar{t}_{i(\sigma)}}\right)+\alpha r_{i(\sigma)}\right) \beta \alpha^{k}$. Since it is never needed to offer a discount greater than $\bar{\alpha}$, this becomes

$$
\alpha^{*}=\operatorname{argmin}_{\alpha \in[0, \bar{\alpha}]} f(\alpha) .
$$

Taking the first derivative of $f$ with respect to $\alpha$ and setting it equal to zero gives

$$
f^{\prime}(\alpha)=\beta \alpha^{k-1}\left((k+1) \alpha r_{i(\sigma)}-k\left(V\left(\sigma_{\bar{t}_{i(\sigma)}}\right)-V\left(\sigma_{t_{i(\sigma)}}\right)\right)=0\right.
$$

which has two solutions: $\alpha^{*}=\frac{k\left(V\left(\sigma_{t_{i(\sigma)}}\right)-V\left(\sigma_{\bar{t}_{i(\sigma)}}\right)\right)}{(k+1) r_{i(\sigma)}}$ and 0. Note that we have $f^{\prime \prime}\left(\alpha^{*}\right)=\left(V\left(\sigma_{t_{i(\sigma)}}\right)-\right.$ $\left.V\left(\sigma_{\bar{t}_{i(\sigma)}}\right)\right)>0$, which implies that the $f$ is convex at $\alpha^{*}$. Hence, the result follows from the observation that $f(0)-f\left(\alpha^{*}\right)=\frac{\beta\left(\frac{k}{2 k+2}\right)^{k}}{k+1}$, which is always positive.

In what follows, we use Lemma 1 to derive analytical results and numerical examples that provide insight into the optimal sequence of discount offers.

We consider the same family of offer acceptance probability functions as in Lemma 1. First, we present an analytical result for the basic, but nontrivial, case $k=1$. Second, we present a numerical analysis for arbitrary value of $k$. We focus on the more interesting settings in which the revenues are high enough (compared to the delivery costs) that optimal discount offers do not result in guaranteed acceptance.

Proposition 3. Consider a PDFP instance with $I=\{1,2\}, T=2, r_{1}=r_{2}, q_{1}, q_{2} \leq 0.5 Q$, $\delta_{1} \neq \delta_{2}$. Let $\bar{t}_{i}$ denote the service day not preferred by customer $i \in I$. Let $P_{i}\left(\alpha, \bar{t}_{i}\right)=\min \left\{\beta_{i} \alpha, 1\right\}$, for $i=1,2$ and for $\alpha \in[0,1]$. Let $\frac{\left.\left(\delta_{1}+\delta_{2}\right)-\max \left\{\delta_{1}, \delta_{2}\right\}\right)}{r} \leq \frac{1}{\beta_{i}}$ for $i=1,2$. If $\beta_{1} \leq \beta_{2}$, then the expected cost of offer sequence $\pi=(2,1)$ is greater than or equal to the expected cost of offer sequence $\bar{\pi}=(1,2)$.

Proof. The assumption that $\frac{\left.\left(\delta_{1}+\delta_{2}\right)-\max \left\{\delta_{1}, \delta_{2}\right\}\right)}{r} \leq \frac{1}{\beta_{i}}$ for $i=1,2$ avoids situations in which an optimal discount offer guarantees acceptance. Note that if $t_{1}=t_{2}$, then the optimal policy is to offer 
no discounts and there is nothing to prove. So without loss of generality, we assume that customer 1 prefers day 1 and customer 2 prefers day 2. For notational convenience, let $V_{A}=2\left(\delta_{1}+\delta_{2}\right)$ be the delivery cost when the customers are served on separate days and $V_{B}=2 \max \left\{\delta_{1}, \delta_{2}\right\}$ be the delivery cost when the customers are served on the same day. By Lemma 1 and the assumption $\frac{\left(V_{A}-V_{B}\right)}{2 r} \geq \frac{1}{\beta_{1}}$, we have $V_{\pi}\left(\sigma^{\emptyset}\right)-V_{\bar{\pi}}\left(\sigma^{\emptyset}\right)=\frac{1}{64 r^{3}} \beta_{1} \beta_{2}\left(V_{A}-V_{B}\right)^{4}\left(\beta_{2}-\beta_{1}\right)$, which is always positive since $\beta_{2} \geq \beta_{1}$. Hence, the result follows.

In Proposition 3, we consider acceptance probability functions $P_{i}\left(\alpha, \bar{t}_{i}\right)=\min \left\{\beta_{i} \alpha^{k}, 1\right\}$ with $k=1$. Using similar arguments, it is easy to prove the same result for $k=0.5$ and $k=2$. However, the complexity of the algebraic operations prohibits a rigorous proof along the same lines for an arbitrary value of $k$. Therefore, we conduct numerical experiments to investigate the validity of Proposition 3 for arbitrary values of $k$. In these experiments, we use $V_{A}=2, V_{B}=1, r=2$, and $\beta_{1}=1$ (using the same notation as in the proof of Proposition 3). In Figure 3, we plot the difference between the minimum expected cost for offer sequence $\pi=(2,1)$ and offer sequence $\bar{\pi}=(1,2)$ for values of $k \in[0.1,2]$ and values of $\left.\beta_{2} \in\{1.25,1.5,2\}\right)$. Based on the results of these experiments, we conjecture that Proposition 3 holds for acceptance probability functions $P_{i}\left(\alpha, \bar{t}_{i}\right)=\min \left\{\beta_{i} \alpha^{k}, 1\right\}$ for any value of $k$.

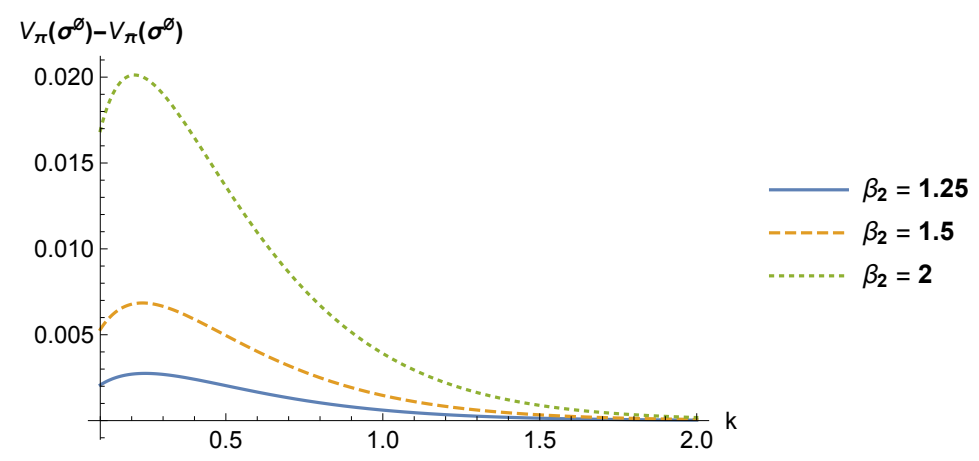

Figure 3: Difference between the expected costs for the offer sequences $\pi$ and $\bar{\pi}$

Next, we consider an orthogonal situation where two customers have identical offer acceptance probabilities and revenues, but they have a different impact on the cost of the delivery plan.

Proposition 4. Consider a PDFP instance $I=\{1, \ldots, n\}, T=2, Q \geq \sum_{\eta \in I} q_{\eta}$ and let $\pi=$ $\left(\pi_{1}, i, j, \pi_{2}\right)$ be an offer sequence such that $d_{i}<d_{j}, r_{i}=r_{j}$ and $t_{i} \neq t_{j}$. If, for $\eta \in\{i, j\}$, $P_{\eta}(\alpha, t)=P(\alpha, t)$ for $t \neq t_{\eta}$, i.e., the customers $i$ and $j$ have equal acceptance probabilities, and $P_{\eta}$ are concave functions of $\alpha$, then the offer sequence $\bar{\pi}=\left\{\pi_{1}, j, i, \pi_{2}\right\}$, which is the same as $\pi$ except that $j$ receives an offer before $i$, results in the expected delivery cost that is never higher than the expected delivery cost induced by $\pi$.

Proof. For notational simplicity we define $\bar{t}_{\eta}=\{1,2\} \backslash t_{\eta}$ as the day not preferred by Customer $\eta$. Let $\sigma$ be an arbitrary state at the $i_{t h}$ level of the state space $\mathcal{S}(\pi)$ and let $\sigma_{t_{i}}$ and $\sigma_{\bar{t}_{i}}$ be the children. Similarly, let $\sigma_{t_{i} t_{j}}$ and $\sigma_{t_{i} \bar{t}_{j}}$ be the children of $\sigma_{t_{i}}$ and let $\sigma_{\bar{t}_{i} t_{j}}$ and $\sigma_{\bar{t}_{i} \bar{t}_{j}}$ be the children of $\sigma_{\bar{t}_{i}}$. We also define $V\left(\sigma_{t_{i} t_{j}}\right)=V_{A}, V\left(\sigma_{t_{i} \bar{t}_{j}}\right)=V_{B}, V\left(\sigma_{\bar{t}_{i} t_{2}}\right)=V_{C}$ and $V\left(\sigma_{\bar{t}_{i} \bar{t}_{j}}\right)=V_{D}$. We denote this (two level) sub-tree as $\mathcal{S}_{\sigma}^{2}(\pi)$ (see Figure 4). 


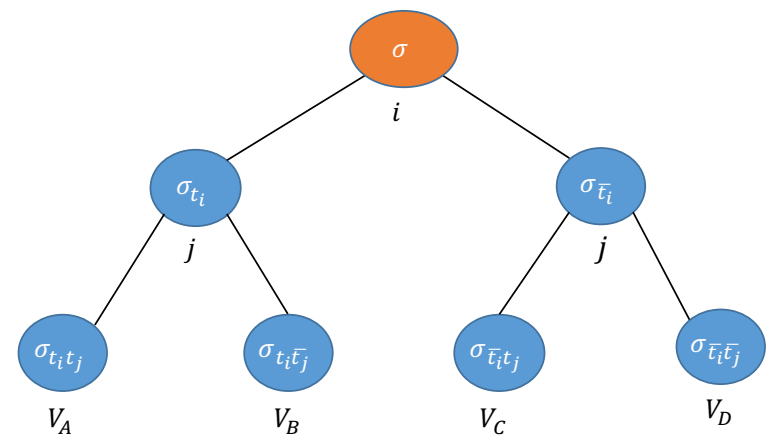

Figure 4: Sub-tree $\mathcal{S}_{\sigma}^{2}(\pi)$

Let $o^{*}$ be the optimal set of offers, $p_{1}=P_{i}\left(\alpha_{\sigma}^{*}, \bar{t}_{i}\right), p_{2}=P_{j}\left(\alpha_{\sigma_{t_{i}}}^{*}, \bar{t}_{j}\right)$ and $p_{3}=P_{j}\left(\alpha_{\sigma_{\bar{t}_{i}}}^{*}, \bar{t}_{j}\right)$. Then the expected cost $V(\sigma)$ of the state $\sigma$ can be calculated as follows.

$$
V(\sigma)=\left(1-p_{1}\right)\left[\left(1-p_{2}\right) V_{A}+p_{2} V_{B}\right]+p_{1}\left[\left(1-p_{3}\right) V_{C}+p_{3} V_{D}\right]+p_{1} \alpha_{\sigma}^{*}+\left(1-p_{1}\right) p_{2} \alpha_{\sigma_{t_{i}}}^{*}+p_{1} p_{3} \alpha_{\bar{t}_{i}}^{*}
$$

Without loss of generality we assume that $t_{i}=t_{1}$ and $t_{j}=t_{2}$. Consider the alternative offer sequence $\hat{\pi}$ that one can derive by switching the order of the customers $i$ and $j$ in $\bar{\pi}$. And let $\hat{\sigma}$ be the $i_{t h}$ level node in $\mathcal{S}(\hat{\pi})$ which matches $\bar{\sigma}$ in $\mathcal{S}(\bar{\pi})$. In Figure 5 , we illustrate $\mathcal{S}_{\hat{\sigma}}^{2}(\hat{\pi})$.

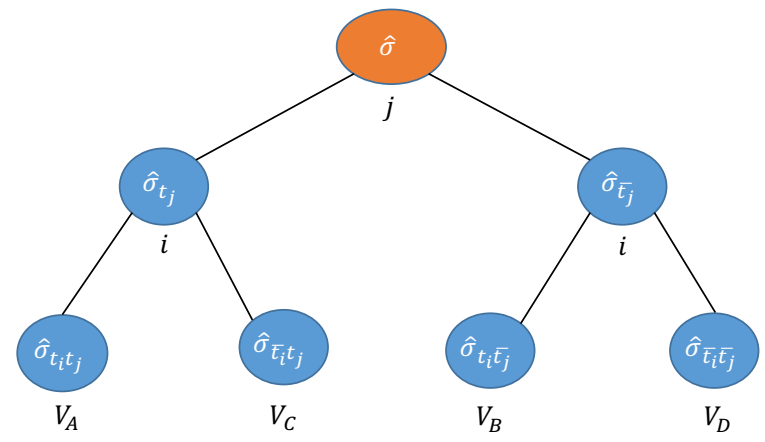

Figure 5: Sub-tree $\mathcal{S}_{\hat{\sigma}}^{2}(\hat{\pi})$

We next show that there exists a set of offers $\hat{o}$ that can achieve the expected value $V(\hat{\sigma})$ that is at least as good as $V(\sigma)$.

Observe $d_{i}<d_{j}$ implies that $V_{B} \leq V_{D}$, because $j$ is served on the same day in both $B$ and $D$, and $i$ is served on the same day as $j$ in $B$. With the same arguments, we also have $V_{C} \leq V_{A}$

We will assume that $\alpha_{\sigma_{t_{i}}}^{*}$ and $\alpha_{\sigma_{\bar{t}_{i}}}^{*}$ are strictly positive, hence $p_{2}$ and $p_{3}$ is strictly positive, since otherwise one can simply consider the offers $\hat{o}_{\hat{\sigma}}=\left(\max \left\{\alpha_{\sigma_{t_{i}}}^{*}, \alpha_{\sigma_{\bar{t}_{i}}}^{*}\right\}, \bar{t}_{j}\right)$ and $\hat{o}_{\sigma_{\bar{t}_{j}}}=\hat{o}_{\bar{t}_{\bar{t}_{j}}}=o_{\sigma}$ that clearly leads to $V(\hat{\sigma})=V(\sigma)$.

Now observe that $p_{2}>0$ implies $V_{B} \leq V_{A}$ and $p_{3}>0$ implies $V_{D} \leq V_{C}$, since $o^{*}$ is an optimal offer set. Then $V_{A} \geq V_{C}$ leads to the following ordering.

$$
V_{A} \geq V_{C} \geq V_{D} \geq V_{B}
$$


Since we have $V_{B} \leq V_{D}$, one should never offer any discount to customer $i$ at state $\hat{\sigma}_{\bar{t}_{j}}$, which gives $V\left(\hat{\sigma}_{\bar{t}_{j}}\right)=V_{B}$. So it is easy to see that as $V_{D}$ gets closer to $V_{B}$ the difference $V(\sigma)-V(\hat{\sigma})$, which we need to show to be always nonnegative to prove our claim, decreases. This is because of the fact that $V(\sigma)$ decrease as $V_{D}$ decreases and $V(\bar{\sigma})$ is not affected by any change in $V_{D}$. So for the sake of simplicity, and without loss of generality, from now on we will assume $V_{B}=V_{D}=0$.

For a state $\bar{\sigma}$ with two children states $\bar{\sigma}_{1}$ and $\bar{\sigma}_{2}$, assuming $V\left(\bar{\sigma}_{1}\right) \leq V\left(\bar{\sigma}_{2}\right)$, we define $\rho_{V\left(\bar{\sigma}_{1}\right) V\left(\bar{\sigma}_{2}\right)} \in$ $[0,1]$ such that $V(\bar{\sigma})=V\left(\bar{\sigma}_{1}\right)+\left(V\left(\bar{\sigma}_{2}\right)-V\left(\bar{\sigma}_{1}\right)\right) \rho_{V\left(\bar{\sigma}_{1}\right) V\left(\bar{\sigma}_{2}\right)}$. Note that for an arbitrary offer acceptance probability function the scalar $\rho_{V\left(\bar{\sigma}_{1}\right) V\left(\bar{\sigma}_{2}\right)}$ is nonincreasing in the difference $\left(V\left(\bar{\sigma}_{2}\right)-V\left(\bar{\sigma}_{1}\right)\right)$. This is because of the fact that the optimal discount is never decreases as $\left(V\left(\bar{\sigma}_{2}\right)-V\left(\bar{\sigma}_{1}\right)\right)$ increases.

For the notational simplicity in our following discussions, we also define $V_{B A}=V\left(\sigma_{t_{i}}\right), V_{B C}=$ $V\left(\sigma_{\bar{t}_{i}}\right), V_{C A}=V\left(\bar{\sigma}_{t_{j}}\right), \rho_{B A}=\rho_{V_{B} V_{A}}, \rho_{B C}=\rho_{V_{B} V_{C}}, \rho=\rho_{V_{B C} V_{B A}}$ and $\hat{\rho}=\rho_{V_{B} V_{C A}}$. Figure 6 illustrates this new notation to provide a quick reference for the reader.

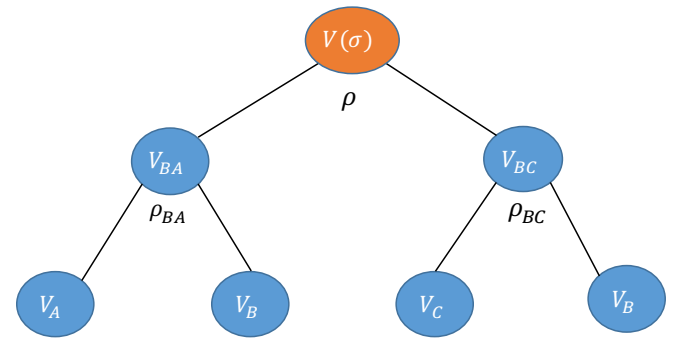

(a) Sub-tree $\mathcal{S}_{\sigma}^{2}(\pi)$

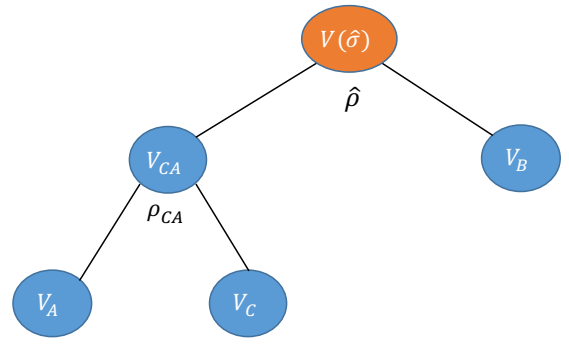

(b) Sub-tree $\mathcal{S}_{\hat{\sigma}}^{2}(\hat{\pi})$

Figure 6: Illustration of the expected cost calculation in sub-trees $\mathcal{S}_{\sigma}^{2}(\pi)$ and $\mathcal{S}_{\hat{\sigma}}^{2}(\hat{\pi})$

Then one can rewrite $V(\sigma)$ and $V(\hat{\sigma})$ as follows.

$$
\begin{aligned}
& V(\sigma)=V_{B C}+\left(V_{B A}-V_{B C}\right) \rho \\
& V(\hat{\sigma})=V_{B}+\left(V_{C A}-V_{B}\right) \hat{\rho}=V_{C A} \hat{\rho},
\end{aligned}
$$

Note that, it must be the case that $\rho \geq \hat{\rho}$, as $V_{B A}-B_{B C} \leq V_{C A}$. To see this, observe that $V_{B A}-V_{B C}$ cannot be larger than $V_{C A}$, simply due to the fact that $V_{B A} \leq V_{C A}$, as $V_{B} \leq V_{C}$. We now show that the difference $V(\sigma)-V(\hat{\sigma}) \geq 0$, as required. By (16) and (17) we have:

$$
\begin{aligned}
V(\sigma)-V(\hat{\sigma}) & =V_{B C}+\left(V_{B A}-V_{B C}\right) \rho-V_{C A} \hat{\rho} \\
& =\left(V_{B C}-V_{C} \hat{\rho}\right)+\left(V_{B A}-V_{B C}\right) \rho-\left(V_{C A}-V_{C}\right) \hat{\rho} \\
& \geq\left(V_{B C}-V_{C} \hat{\rho}\right)+\left(V_{B A}-V_{B C}-V_{C A}+V_{C}\right) \rho
\end{aligned}
$$

So, to show $V(\sigma)-V(\hat{\sigma}) \geq 0$ it is enough to demonstrate that the terms $\left(V_{B C}-V_{C} \hat{\rho}\right)$ and $\left(V_{B A}-V_{B C}-V_{C A}+V_{C}\right)$ are both nonnegative.

- Since we have $V_{B C}=V_{B}+\left(V_{C}-V_{B}\right) \rho_{B C}=V_{C} \rho_{B C}$, we can rewrite the former as $V_{C} \rho_{B C}-V_{C} \hat{\rho}$. Not that we have $\left(V_{C}-V_{B}\right) \leq V_{C}+\left(V_{A}-V_{C}\right) \rho_{C A}=V_{C A}=\left(V_{C A}-V_{B}\right)$, so it must be the case that $\rho_{B C} \geq \hat{\rho}$, which implies $V_{B C}-V_{C} \hat{\rho} \geq 0$, as needed.

- For the latter, recall that we assume the offer acceptance probabilities are concave functions of discount offers, which implies $V_{B A} \geq V_{B C}+\left(V_{C A}-V_{C}\right)$. To see this, observe that the concavity of the acceptance probabilities imply the diminishing returns for increasing discount offers, which makes it more advantageous to make two small offers instead of a single one. 
Hence, the result follows.

Proposition 4 indicates that, given that the customers are "similar" to each other respect to the expected cost of service day changes, it is (most of the time) a "good" strategy to first make offers to those customers with higher impact on the delivery cost. As an important theoretical insight about the complexity of the problem at hand, here we want to mention that all the assumptions we make in Proportion 4 regarding to the offer acceptance probabilities and the delivery day preferences are not just sufficient but necessary. Below we present numerical examples to prove this claim.

We first consider the case with non-concave probability acceptance functions. Using the notation defined in the proof of Proposition 4 and assuming $V_{A}=1, V_{B}=0, V_{D}=0, P_{\eta}\left(\alpha, \bar{t}_{\eta}\right)=$ $\min \left\{1,1.5 \alpha^{k}\right\}$ and $r_{i}=r_{j}=1$, we plot the difference $V(\sigma)-V(\bar{\sigma})$, for $k \in\{0.5,1.5,2,2.5\}$ and $V_{C} \in[0,1]$ in Figure 7. As we see in the Figure, when the acceptance probabilities are convex in discount offers $\alpha$ (i.e., $k>1$ ), there can be cases where making an offer to customer $j$ (who has a higher impact on the delivery cost) before the customer $i$ would result in a slightly worse expected cost for the whole problem. However, note that the cases where such a results occur is limited to $V_{C}$ values that are quite close to $V_{B}$ (cases where the impact of the customer $j$ 's service time has a smaller impact on the overall expected cost) and the magnitude of the difference is relatively smaller, compared to the cases where making the switch improves the expected cost (i.e., $V(\sigma)-V(\bar{\sigma})>0)$.
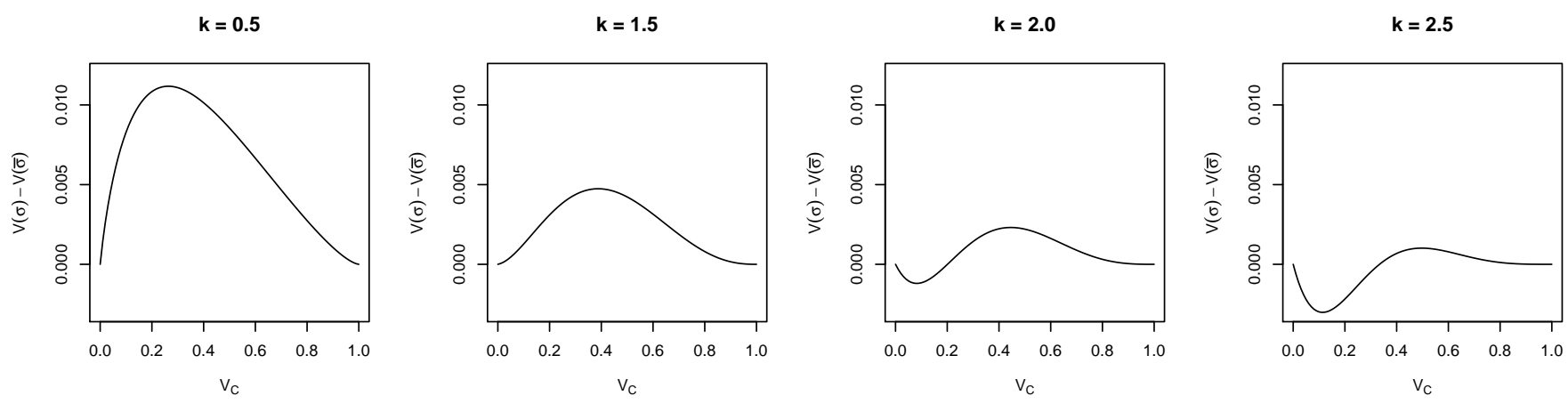

Figure 7: Expected cost differences for the offer sequences $\pi$ and $\bar{\pi}$.

We also investigate the cases where the proposed ordering in Proposition 4 might not improve the expected cost, when the customers offer acceptance probabilities are concave but both customers prefer the same day for service (i.e. $t_{i}=t_{j}$ ). In this case we redefine the following notation introduced in the proof Proposition $4 V\left(\sigma_{\bar{t}_{i} t_{j}}\right)=V_{A}, V\left(\sigma_{\bar{t}_{i} \bar{t}_{j}}\right)=V_{B}, V\left(\sigma_{t_{i} t_{j}}\right)=V_{C}, V\left(\sigma_{t_{i} \bar{t}_{j}}\right)=V_{D}$. Figure 8 illustrates the sub-trees $\mathcal{S}_{\sigma}^{2}(\pi)$ and $\mathcal{S}_{\hat{\sigma}}^{2}(\hat{\pi})$, for the updated notation. Like in the proof of Proposition 4, we will assume that $\alpha_{\sigma_{t_{i}}}^{*}$ and $\alpha_{\sigma_{\bar{t}_{i}}}^{*}$ are strictly positive, hence $p_{2}$ and $p_{3}$ is strictly positive, since otherwise one can simply consider the offers $\hat{o}_{\hat{\sigma}}=\left(\max \left\{\alpha_{\sigma_{t_{i}}}^{*}, \alpha_{\sigma_{\bar{t}_{i}}}^{*}\right\}, \bar{t}_{j}\right)$ and $\hat{o}_{{\overline{t_{j}}}_{j}}=$ $\hat{o}_{\bar{t}_{j}}=o_{\sigma}$ that clearly leads to $V(\hat{\sigma})=V(\sigma)$. In this case, $p_{2}>0$ implies $V_{D} \leq V_{C}$ and $p_{3}>0$ implies $V_{B} \leq V_{A}$, since $o^{*}$ is an optimal offer set. Then $V_{A} \geq V_{C}$ leads to the following ordering.

$$
V_{A} \geq V_{C} \geq V_{D} \geq V_{B}
$$




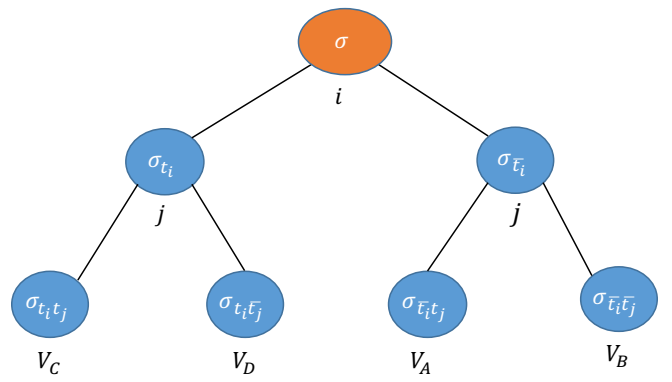

(a) Sub-tree $\mathcal{S}_{\sigma}^{2}(\pi)$

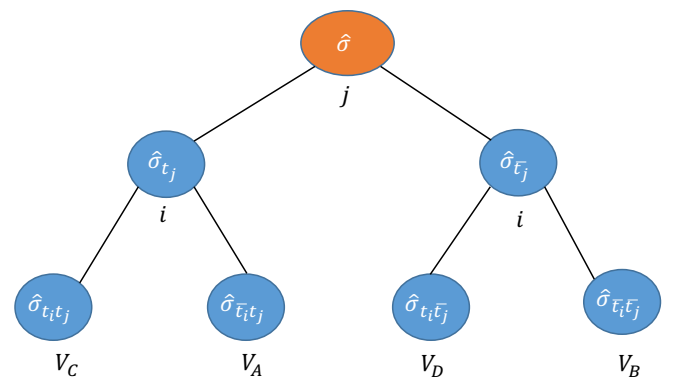

(b) Sub-tree $\mathcal{S}_{\hat{\sigma}}^{2}(\hat{\pi})$

Figure 8: Illustration of the expected cost calculation in sub-trees $\mathcal{S}_{\sigma}^{2}(\pi)$ and $\mathcal{S}_{\hat{\sigma}}^{2}(\hat{\pi})$

Since we have $V_{C} \leq V_{A}$, one should never offer any discount to customer $i$ at state $\hat{\sigma}_{t_{j}}$, which gives $V\left(\hat{\sigma}_{t_{j}}\right)=V_{C}$. So it is easy to see that as $V_{A}$ gets closer to $V_{C}$ the difference $V(\sigma)-V(\hat{\sigma})$, which we want to do to produce a counter example. This is because of the fact that $V(\sigma)$ decreases as $V_{A}$ decreases and $V(\bar{\sigma})$ is not affected by any change in $V_{A}$. So for the sake of simplicity, and without loss of generality, from now on we will assume $V_{A}=V_{C}$ and $V_{B}=0$, as before. With this modifications, the expected cost calculations are illustrated in Figure 9.

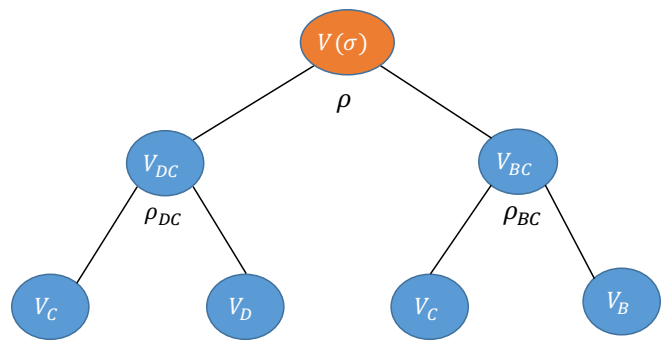

(a) Sub-tree $\mathcal{S}_{\sigma}^{2}(\pi)$

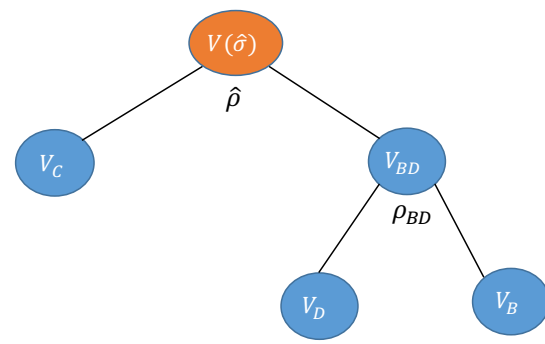

(b) Sub-tree $\mathcal{S}_{\hat{\sigma}}^{2}(\hat{\pi})$

Figure 9: Illustration of the expected cost calculation in sub-trees $\mathcal{S}_{\sigma}^{2}(\pi)$ and $\mathcal{S}_{\hat{\sigma}}^{2}(\hat{\pi})$

As before, we will consider a setting where $r_{i}=r_{j}=1$ and $P_{\eta}\left(\alpha, \bar{t}_{\eta}\right)=\min \left\{1,1.5 \alpha^{k}\right\}$. In this case we consider $V_{A}=1, V_{B}=0, V_{C}=1$ and plot the difference $V(\sigma)-V(\bar{\sigma})$, for $k \in$ $\{0.25,0.75,1.25\}$ and $V_{D} \in[0,1]$, in Figure 10. As we can see from the graphs, for small $k$ values $(k=0.3)$, there can be cases where making an offer to customer $i$ before the customer $j$ is preferable. However, from the practical point of view we can also observe that when such cases occur the difference is relatively small.

\subsection{An enhanced dynamic programming algorithm}

The state space of the dynamic program introduced in the previous section grows rapidly when the number of customers and the number of days in the planning period increases. As a result, the dynamic program can only be used to solve relatively small instances, i.e., instances with no more than 15 customers.

However, properties of certain offer sequences can be exploited to significantly enhance the computational efficiency of the dynamic program. We focus on the sequence in which offers to 

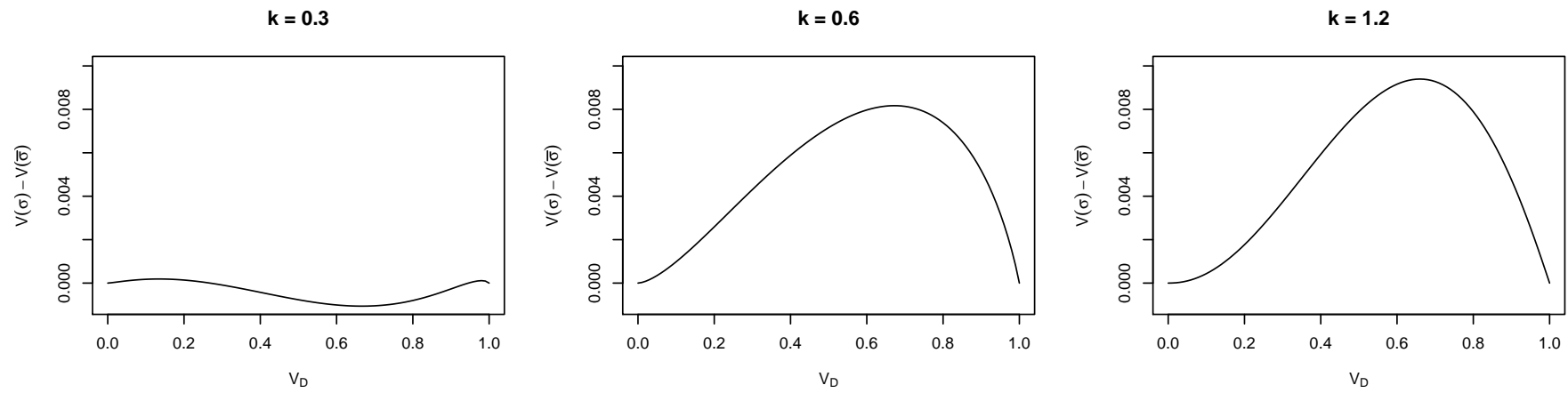

Figure 10: Expected cost differences for the offer sequences $\pi$ and $\bar{\pi}$.

customers are made in non-increasing order of their distance to the depot. Recall that the results in the previous section suggest that this offer sequence is likely to produce high-quality solutions.

To establish the computational benefits of using this specific offer sequence, we first define the notions of a dense and a feasible full state.

Definition 3. A state $\sigma$ is said to be dense if each one of its lists contains at least one customer, i.e., $\left|L_{t}^{\sigma}\right| \geq 1$ for $t=1, \ldots, T$. A dense state $\sigma$ is called feasible, if the remaining vehicle capacity is sufficient to accommodate the demand of all remaining customers on their preferred day, i.e., $Q \geq q\left(L_{t}^{\sigma}\right)+\sum_{i \in I \backslash I(\sigma): i_{t}=t} q_{i}$ for $t=1, \ldots, T$.

The advantage of using this offer sequence comes from the following observation.

Observation 1. Let $\sigma$ be a feasible dense state when making offers to customer in non-increasing order of their distance to the depot, then $V(\sigma)=\sum_{t=1}^{T} 2 \delta\left(L_{t}^{\sigma}\right)$

The observation follows from the fact that when making offers to customers in non-increasing order of their distance to the depot, for a dense state $\sigma$, we have that $\delta_{i} \leq \delta\left(L_{t}^{\sigma}\right)$, for all $t \in 1, \ldots, T$, where $i$ is the customer being consider in state $\sigma$.

As a consequence, we can refine Algorithm 1, as shown in Algorithm 3, by modifying the condition in Line 5. This reduces the state space (search tree) significantly, but we still obtain the minimum expected cost for the sequence.

\section{$3.3 \quad$ A greedy heuristic}

To assess the value of optimizing the offer sequence and the offer value, we also implement a greedy heuristic. The greedy heuristic uses a fixed offer value (e.g., $\alpha=0.1$ ) and iteratively seeks to reduce the total cost starting from the solution in which all orders are delivered on their preferred day. More specifically, in each iteration, the greedy heuristic finds the day for which "moving" the furthest customer results in the largest cost reduction, i.e., the day with the largest difference between the distance to the furthest and the second furthest customer, finds the best day to move that customer to, i.e., the day for which the furthest customer is furthest away from the depot, and, if the cost reduction is greater that the cost of the discount offer, offers the customer a discount to accept a delivery on a different day. Regardless of whether or not the customer accepts the offer, 


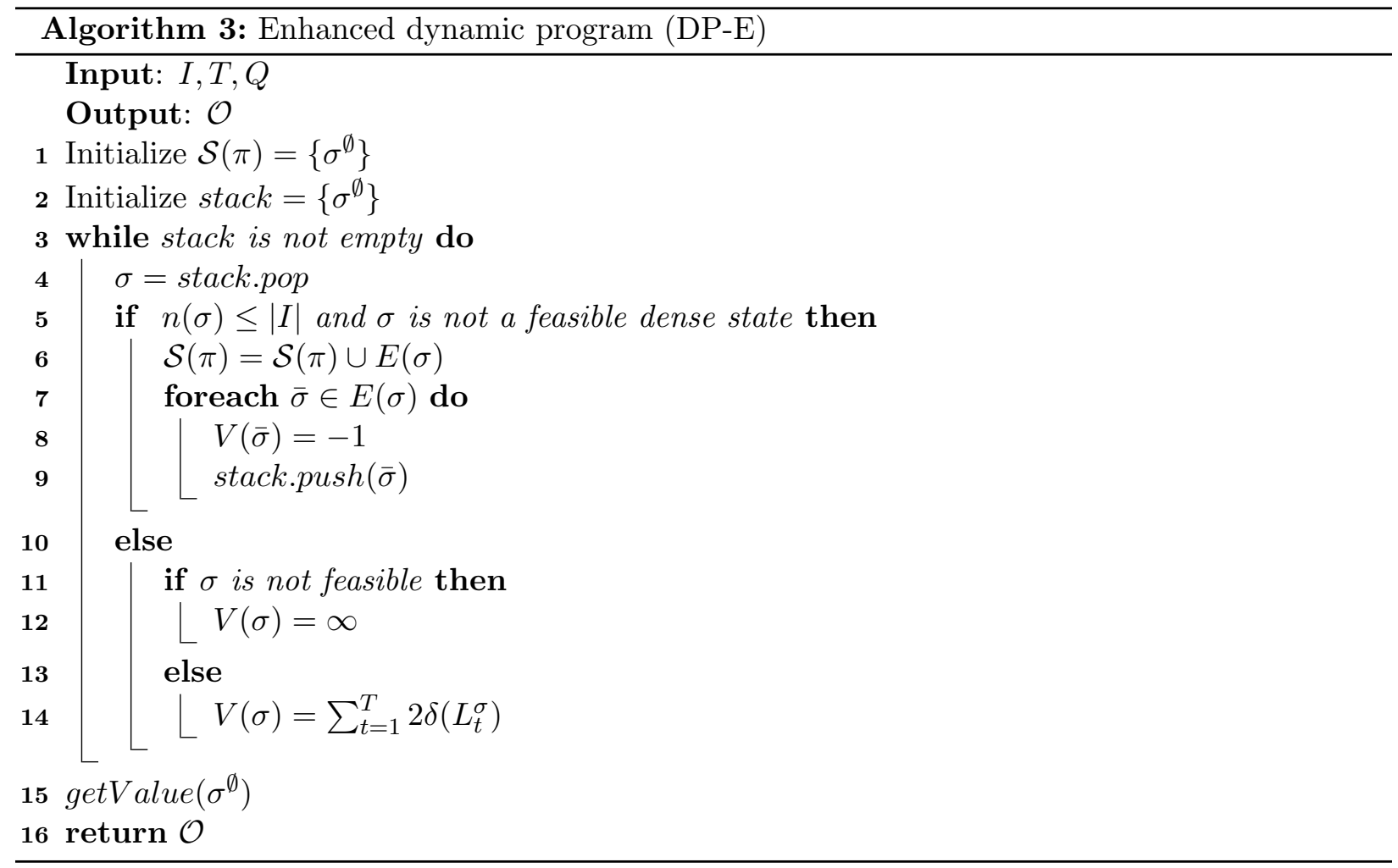

that customer cannot receive any further offers. The heuristic terminates if no further offers can be made (i.e., the furthest customer on each day has already received an offer) or no further cost reduction is possible. For details, see Algorithm 4. To determine the expected cost of the solution produced by the greedy heuristic, we run the heuristic a 1,000 times and take the average as the expected cost.

\section{Computational study}

In order to evaluate the potential benefits of offering discounts in return for delivery flexibility, and the efficacy of our solution approach, we have conducted a comprehensive numerical study. In the following sections, we discuss the goals of the study, the instances used, the analysis of the results, and the insights obtained.

\subsection{Experimental design}

The goals of the numerical study are to answer, among others, the following questions:

- What are the benefits of offering discounts in return for delivery flexibility in different settings?

- How do the temporal and spatial distribution of the delivery locations affect the benefits of pricing for delivery flexibility?

- How do the customer acceptance probability functions affect the benefits of pricing for delivery flexibility? 


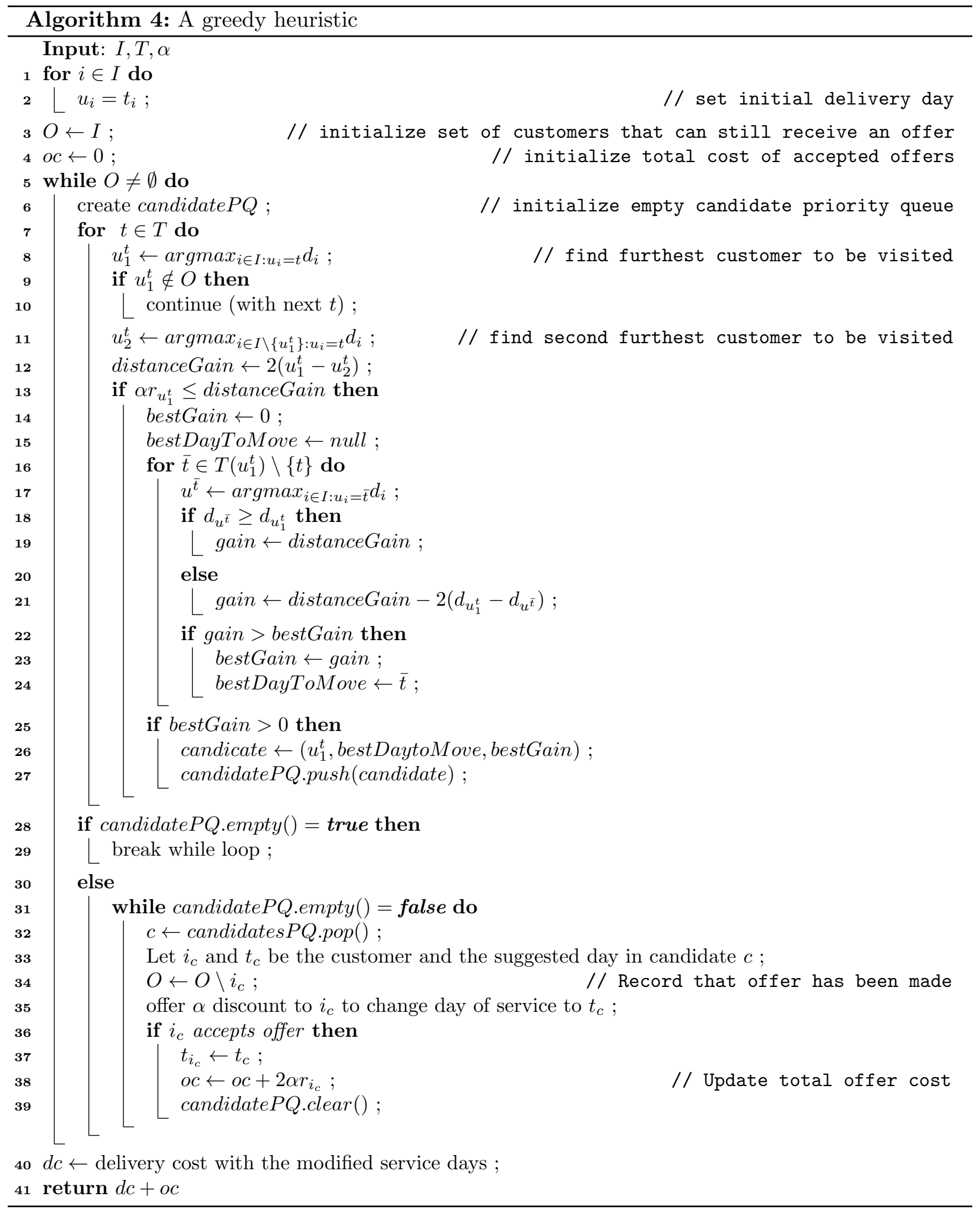


- How does the ratio of revenue earned and delivery cost affect the benefits of pricing for delivery flexibility?

- How does the ratio of expected daily demand and daily delivery capacity affect the benefits of pricing for delivery flexibility?

- What is the value of optimizing the discount offers (compared to using a simple greedy rule)?

- What is the quality of the solutions obtained when we make offers to customer in nonincreasing order of their distance to the depot?

To try to answer these questions, we conducted six experiments. These experiments seek to establish the impact of (1) the average number of daily deliveries, (2) the temporal distribution of the deliveries - across the days in the planning horizon, (3) the spatial distribution of the deliveries - relative to the location of the depot, (4) the offer acceptance probability functions, (5) the vehicle capacity, and (6) the optimization of the (individual) discounts.

All instances used in our computational experiments have a planning horizon of five days $(T=$ $5)$, delivery locations in the line segment $(0,1]$, and the depot located at 0 . We vary the number of orders from 15 to 40; more specifically $n \in\{15,20,25,30,35,40\}$. The weight of an order $q_{i}$ is drawn uniform randomly from $\{1,2,3\}$. The remaining parameters, i.e., location distribution, preferred day distribution, acceptance probability function, revenue, and vehicle capacity depend on the specific experiment. In each of our experiments and for each number of orders, we randomly generate five instances, and use the average over these five instances in our analyses.

The additional parameters of our base case are:

- location distribution: $d_{i}$ uniform random in $(0,1]$;

- preferred day distribution: $t_{i}$ uniform random in $\{1,2,3,4,5\}$;

- offer acceptance probability function: $P_{i}\left(\alpha, t_{i}\right)=\min \left\{1, \beta_{i}^{t} \alpha^{k}\right\}$, where $k=0.25, \beta_{i}$ is uniform random in $[1,2]$, and

$$
\beta_{i}^{t}=\left\{\begin{array}{l}
0.9 \beta_{i}, \text { if } t_{i}-t=1 \\
0.81 \beta_{i}, \text { if } t_{i}-t=2 \\
0.81 \beta_{i}, \text { if } t_{i}-t=-1 \\
0.729 \beta_{i}, \text { if } t_{i}-t=-2 \\
0, \text { otherwise }
\end{array}\right.
$$

Note that the value $\beta_{i}^{t}$ adjusts the acceptance probabilities so that offers for earlier delivery and delivery closer to the preferred day are more likely to get accepted.

- revenue: $r_{i}=r d_{i}$; and

- vehicle capacity: $Q$ not limiting.

In the first set of experiments (E1), we analyze the benefits of pricing for delivery flexibility in the base case, using both the greedy heuristic and the enhanced dynamic programming algorithm when making offers to customers in non-increasing order of their distance to the depot. In the second set of experiments (E2), we investigate the impact of the temporal distribution of the customers. To do so, we generate additional instances using a triangular distribution (with peak 
probability on day 3) to determine the preferred delivery day and an inverse triangular distribution (with peak probabilities on days 1 and 5) to determine the preferred delivery day. In the third set of experiments (E3), we explore the impact of the spatial distribution of customers. We consider the following three probability density functions for the location distributions: $f_{1}(x)=2-2 x$ (so that customers are mostly located close to the depot), $f_{2}(x)=1$ (so that customers are uniformly distributed), and $f_{3}(x)=2 x$ (so that customers are mostly located far away from the depot). In the fourth set of experiments (E4), we consider different offer acceptance probability functions. Specifically, we vary the parameter $k(k=0.125,0.25$, and 0.5$)$ and we introduce threshold offer acceptance probability function

$$
P_{i}(\alpha, t)=\left\{\begin{array}{l}
0, \text { if } \beta_{i}^{t}=0 \text { or } \alpha<\sqrt[k]{\frac{1}{\beta_{i}^{t}}} \\
1, \text { o.w. }
\end{array}\right.
$$

In Figure 11, we illustrate these offer acceptance probability functions for a randomly picked customer $i$ (with $\beta_{i}=1.5$ ), where part (a) shows the offer acceptance probability functions for service a day late and part (b) shows the offer acceptance probability functions for service a day early. In the

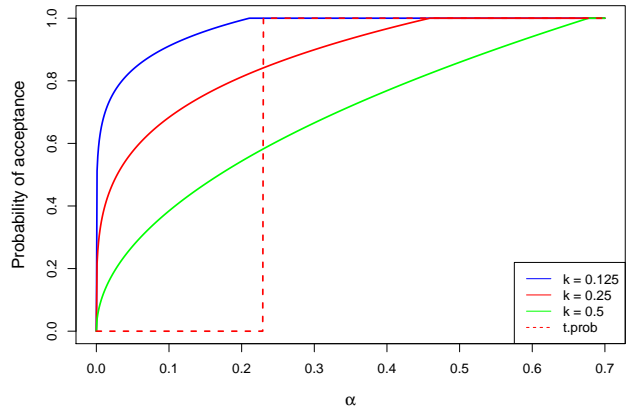

(a) Service one day late

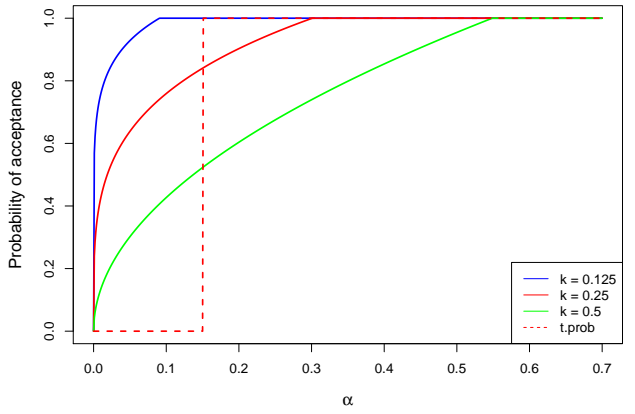

(b) Service one day early

Figure 11: Customer offer acceptance probabilities

fifth set of experiments (E5), we explore different ratios of revenue and cost by varying parameter $r(r=0.9,1,1.1,1.2,1.3)$. Finally, in the sixth set of experiments (E6), we consider the impact of different vehicle capacities. Specifically, we set $Q=v \bar{q}$, where $\bar{q}=\frac{2 n}{5}$ is the expected demand on a day (assuming $n$ customers and 5 days), for $v \in\{1.25,1.5,2\}$ (i.e., we consider instances with vehicle capacities that are 1.25, 1.5, and 2 times larger than the expected delivery quantity on a day).

\subsection{Distance-based offer sequence}

Unfortunately, because the state space of our dynamic programming algorithm grows exponentially with the number of customers, only small instances can be solved optimally. However, the analytical results suggest that making offers to customers in non-increasing order of their distance to the depot should produce high-quality solutions and our enhanced dynamic programming algorithm for that specific offer sequence is far more efficient. To validate the belief that the "distance-based offer sequence" is quite effective, we conduct the following experiment. We take five instances with 11, 12, 
13, 14, and 15 customers, customers' preferred days uniformly distributed across a 5-day planning horizon, and remaining instance characteristics as in the base case. For each of these instances, we randomly chose 100 different offer sequences and compute the expected cost reduction for each of them as well as for the distance-based offer sequence. The detailed results of this experiments can be found in Table 2 in the appendix, but the critical information is presented in Figure 12, where we plot the expected cost reductions for each offer sequence for each instance, highlighting in red the cost reduction for the distance-based offer sequence in red and the number of offer sequences that perform worse.

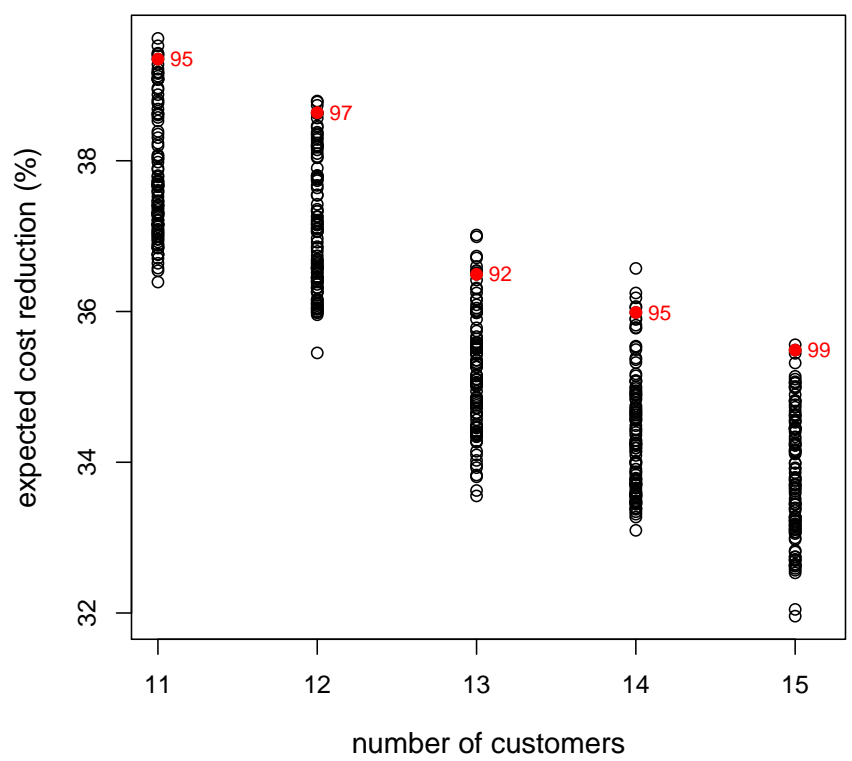

Figure 12: Impact of the offer sequence on the expected cost reduction

We observe that the offer sequence has a non-negligible impact on the cost savings (up to $3.6 \%$ for these instances), and that the distance-based offer sequence performs extremely well, i.e., results in large cost reductions. Because of its computational advantages, we therefore use the distance-based offer sequence in all our further experiments.

We also observe that the cost reduction decreases when the number of customers in the instance increases, which is to be expected as consolidation opportunities naturally arise when the number of customers increases. This aspect will be covered in more depth as part of the analysis of the results of the comprehensive computational study presented next.

\subsection{Analysis}

Detailed results of the first set of experiments (E1) can be found in Table 3 in the appendix, but the critical information is captured in Figure 13. In Part (a), we show the expected cost reductions for the base case obtained by the greedy heuristic (GS) for different fixed offer values $(\alpha=0.05,0.1$, 
and 0.2) and the enhanced dynamic programming algorithm for the distance-based order sequence (E-DP). In Part (b), we show the cost of the discounts offered as a fraction of the total cost.

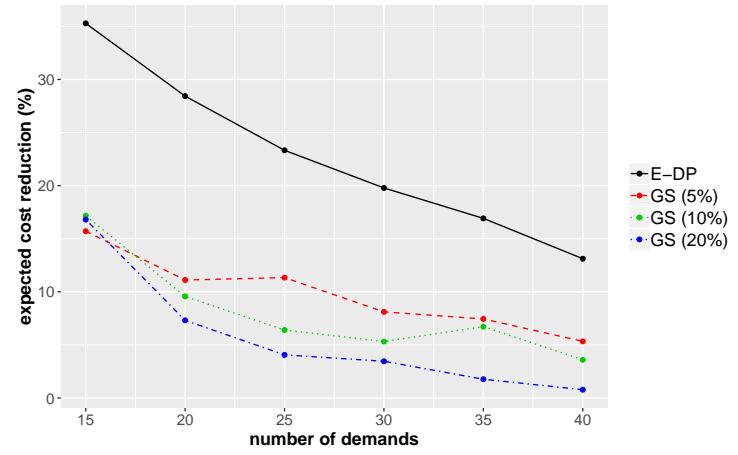

(a) Cost savings for E-DP and GS solutions

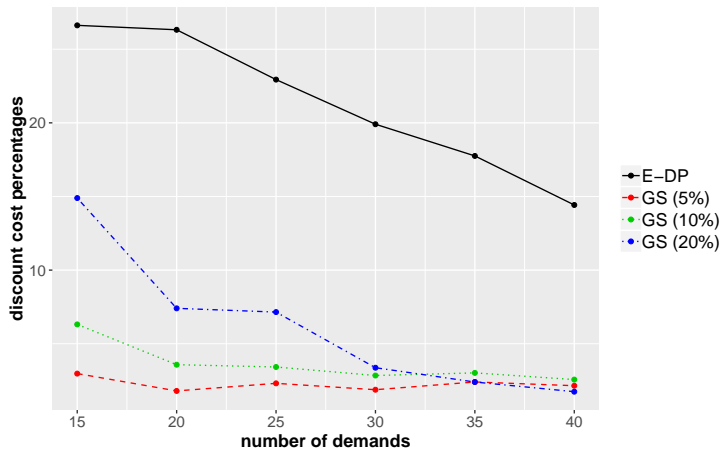

(b) Share of the discount cost in these solutions

Figure 13: E1 results.

The graph in Part (a) clearly demonstrates the significant benefit of offering discounts in return for service time flexibility. For these instances, cost reductions of more than $35 \%$ can be seen when the average number of orders per day is small and consolidation opportunities do not naturally emerge. As the average number of orders per day increases, the cost reductions get smaller as consolidation opportunities start to emerge naturally. However, even for the instances with the largest average number of orders per day, the cost reduction is more than $10 \%$. We also observe that using the enhanced dynamic programming algorithm for the distance-based offer sequence solutions clear outperforms the greedy heuristic and provides about two times more cost savings, regardless of the fixed offer value, which indicates the importance of optimizing the discount offer. The graph in Part (b), shows even more clearly that when the average number of orders per day increases, there are fewer opportunities to obtain cost savings by incentivizing customers to accept a delivery on a day other than their preferred day, because the majority of the total cost are delivery costs and only a small portion is offer discount costs.

The results of these experiments clearly show the significance of customer density (i.e., the number of orders that have to be delivered on a particular day) on the benefits of offering discounts in return for delivery flexibility. Another aspect that can have an impact is the temporal distribution of the customers' preferred delivery days, which is explored in our second set of experiments. Detailed results of these experiments (E2) can be found in Table 4 in the appendix, but the critical information is captured in Figure 14, where we show the expected cost reductions, in Part (a), and the discount cost percentages, in Part (b), for a uniform, a triangular and an inverse triangular distribution of the preferred delivery days.

Interestingly, even for the triangular and inverse triangular distributions of preferred delivery days, which should lead to more natural opportunities for consolidation due to higher customer density on certain days, the expected cost reductions remain quite significant. For the triangular distribution, we see that the expected cost reductions are even higher than the expected cost reductions for the uniform distribution when the total number of customers increases. This may be explained by the fact that when many customers have the third day as their preferred day, this day has high customer density, but it implies that the other days (i.e., the first two and the last two) have low customer densities, and we know that offering discounts is especially beneficial when 


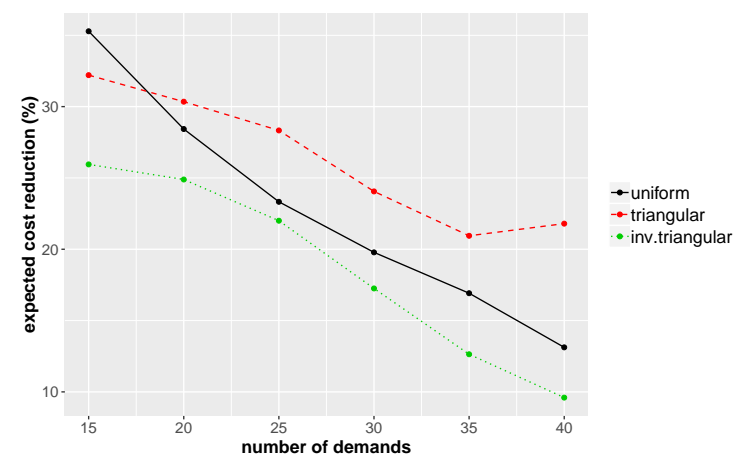

(a) Cost savings for E-DP solutions

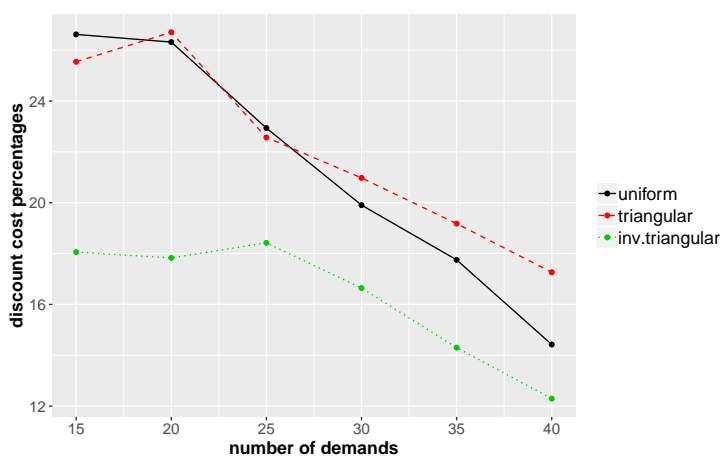

(b) Share of the discount cost in these solutions

Figure 14: E2 results

customer densities are low. (With the inverse triangular distribution this effect is not as strong because there are two peaks rather than one.) This observation may also explain why the fraction of offer discount cost is high for the triangular distribution: the opportunities for delivery cost savings are high and thus many discount offers and/or high discount offers are made.

In addition to the distribution of the customers' preferred delivery day, their spatial distribution, i.e., the distribution of their delivery locations, also impacts the delivery costs and the benefit of offering discounts for service time flexibility, which we explore in our third set of experiments. Detailed results of these experiments (E3) can be found in Table 5 in the appendix, but the critical information is captured in Figure 15, where we show the expected cost reductions, in Part (a), and the discount cost percentages, in Part (b), for different spatial distributions of customer locations (i.e., $f_{1}(x)=2-2 x-$ so that customers are mostly located close to the depot, $f_{2}(x)=1-$ so that customers are uniformly distributed, and $f_{3}(x)=2 x$ - so that customers are mostly located far away from the depot).

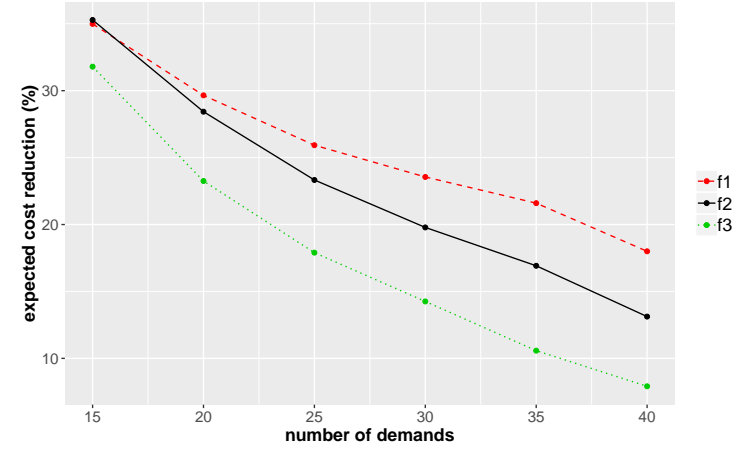

(a) Cost savings for E-DP solutions

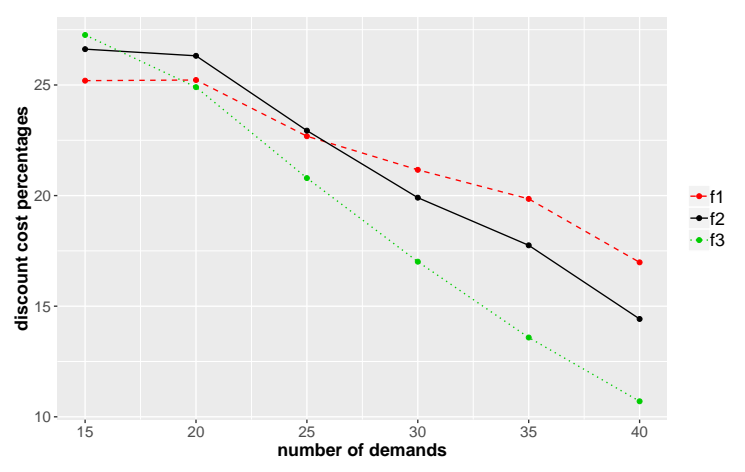

(b) Share of the discount cost in E-DP solutions

Figure 15: E3 results

The fact that the expected cost reduction is higher when customers are mostly located close to the depot (spatial distribution $f_{1}(x)$ can be explained as follows. When delivery locations are mostly close to the depot, but there are a few delivery locations far away, it is easy to identify the delivery day changes that lead to significant cost reductions (by serving far away locations 
on the same day). When delivery locations are mostly far away from the depot, fewer of these opportunities exist and they are harder to identify.

The willingness of customers to accept a discount offer to allow a delivery on a day other than their preferred day will have an impact on the benefits of offering such discounts, which is the focus of our fourth set of experiments. Detailed results of these experiments (E4) can be found in Table 6 in the appendix, but the critical information is captured in Figure 16, where we show the expected cost reductions, Part (a), and the discount costs, Part (b), for different offer acceptance probability functions, including a threshold acceptance probability function (where smaller values of the parameter $k$ imply that a customer is more likely to accept a discount offer).

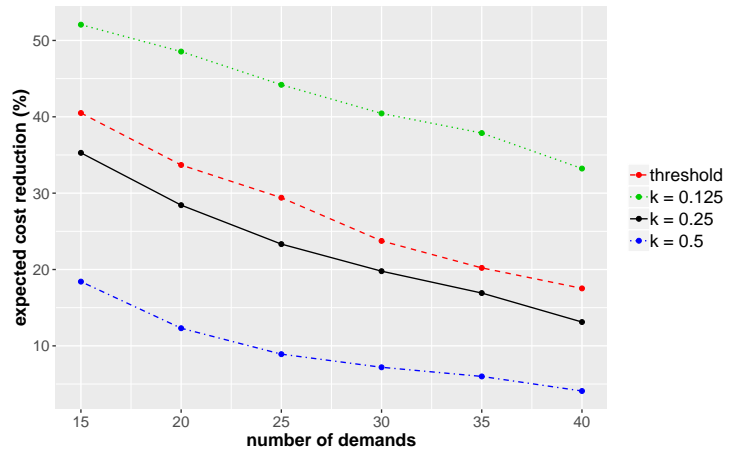

(a) Cost savings for E-DP solutions

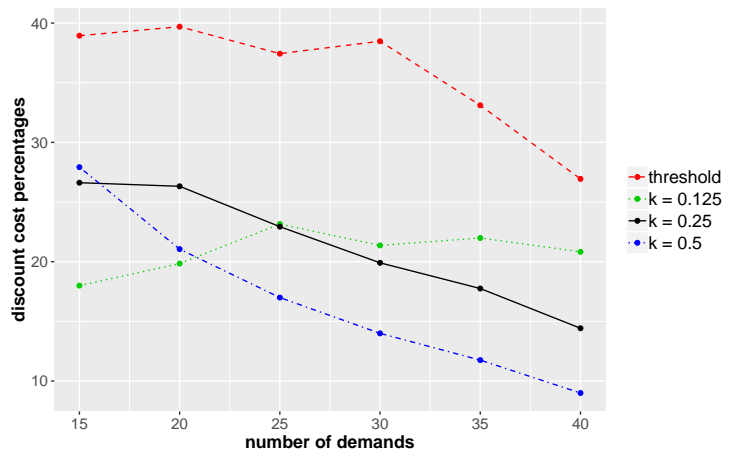

(b) Share of the discount cost in these solutions

Figure 16: E4 results

As expected, we see that the benefit of offering discounts in return for delivery flexibility increases as the customers are more likely to accept these offers. Interestingly, the threshold acceptance probability function, which is defined to closely follow the acceptance probability function with parameter $k=0.25$ (see Figure 11), and is mostly dominated by that function, provides higher expected cost reductions. The graph with discount cost percentages, i.e., Part (n), suggests that with the threshold acceptance probability function significantly more discount offers are accepted (and possibly made) than with the other acceptance probability functions. This is not surprising because there is no uncertainty. Any discount offer made will be such that it is accepted (and at the lowest discount for which this will happen).

The revenue generated by an order also affects the benefits of offering discounts for delivery flexibility. When the costs of delivery are greater than the costs of discounts, the benefits of of offering discounts for delivery flexibility should be more pronounced. The fifth set of experiments seeks to validate this intuition. Detailed results of these experiments (E5) can be found in Table 7 in the appendix, but the critical information is captured in Figure 17, where we show the expected cost reductions, Part (a), and the discount costs, Part (b), for different revenue multipliers.

The results support the intuition. However, it is interesting to observe that the difference in expected cost reductions across the various values of $r$ is quite small. Even if the revenue multiplier is increased by almost 50\% (i.e., from 0.9 to 1.3), the change in expected cost reduction is less than $9 \%$. This suggests that the benefits of offering discounts in return for service time flexibility is quite robust against differences in order revenues.

In our last set of experiments, we focus on the impact of vehicle capacity on the benefit of offering discounts for delivery flexibility. Detailed results of these experiments can be found in 


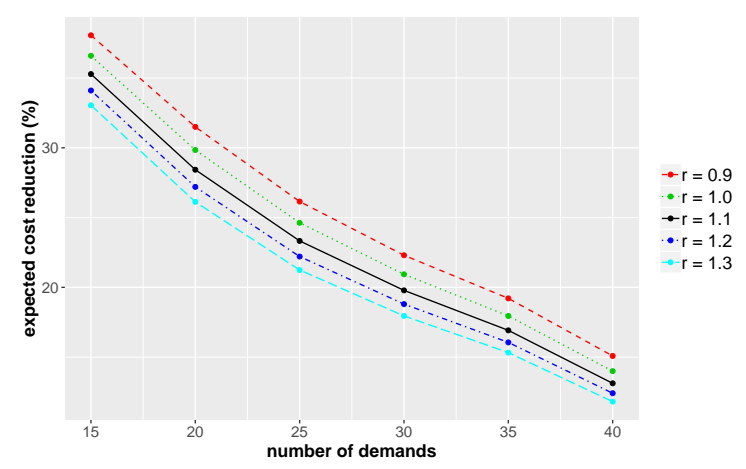

(a) Cost savings for E-DP solutions

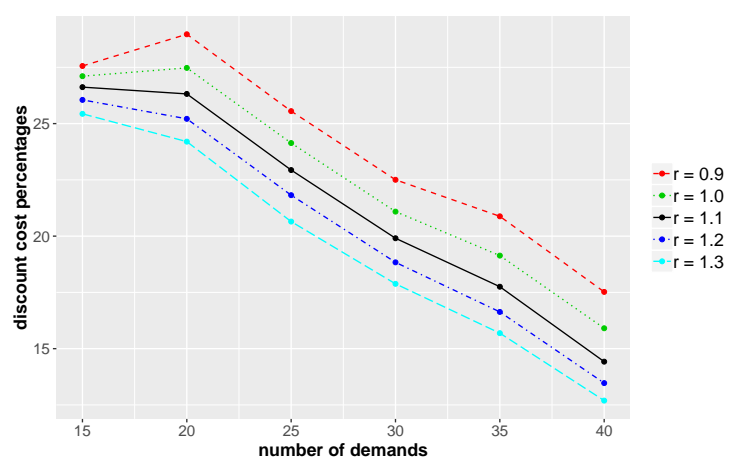

(b) Share of the discount cost in these solutions

Figure 17: E5 results

Table 8 in the appendix, but the critical information is captured in Figure 18. In Part (a), we present the expected cost reduction for different vehicle capacities $\left(Q=v \frac{\sum_{i=1}^{n} q_{i}}{T}\right.$ for $v=1.25,1.5$, and 2). To get the cost of a solution without offering discounts for delivery flexibility, we check if an instance is feasible, i.e., whether the vehicle capacity is sufficient to deliver all orders on their preferred day, and, if not, we solve a knapsack problem to determine a set of orders of minimum total revenue to "reject" so as to ensure that a feasible solution exists. The rejected revenue is added to the distribution cost (as an opportunity cost) to obtain the cost for the instance.

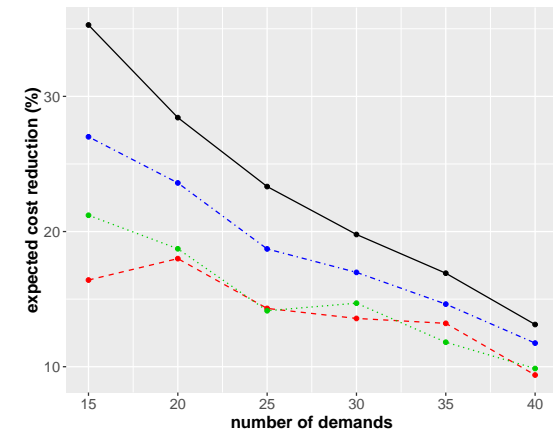

(a) Cost savings for E-DP solutions

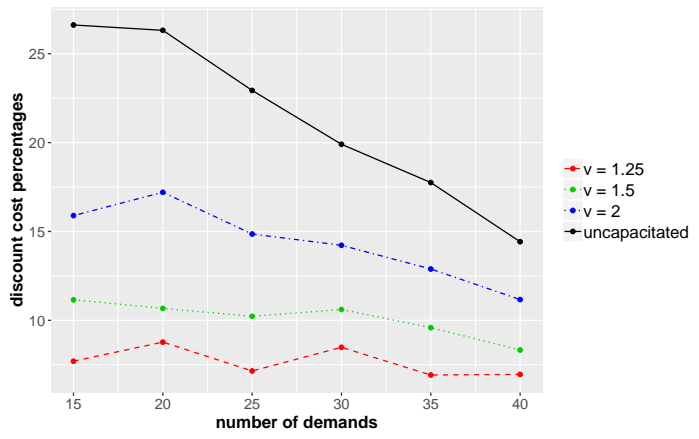

(b) Share of the discount cost these solutions

Figure 18: E6 results

As expected, vehicle capacity has a significant impact on the benefit of offering discounts for delivery flexibility. We note that due to the sequential nature of the offering process, an offer is made only if sufficient capacity is available to accommodate the order on the offered day, i.e., we are not considering "swaps" of orders. To reach high levels of expected cost reduction, a significant amount of excess capacity $\left(Q-\frac{\sum_{i=1}^{n} q_{i}}{T}\right)$ is necessary. However, even when excess capacity is small, the benefits of offering discounts for delivery flexibility are still worthwhile. 


\section{$5 \quad$ Final remarks}

We have studied a variant of the multi-period vehicle routing problem, in which a service provider offers a discount to customer in exchange for delivery flexibility. We have established properties and empirical insights regarding the intricate and complex relation between the benefits from additional delivery flexibility, the discounts offered to customers to gain this additional delivery flexibility, and the likelihood of acceptance of the discount offers by customers. The computational experiments, using an exact dynamic programming algorithm for a particular offer sequence show that, depending on the setting, cost savings exceeding $30 \%$ can be achieved.

The computational experiments have focused on instances in which delivery locations are on the line segment $[0,1]$ with the depot located at 0 . This was done to simplify interpretation of the resulting solutions. We believe that many of the insights obtained will extend to more complex geometries, but, of course, that believe has to be validated. We plan to do so in the near future.

\section{References}

Niels Agatz, Ann Campbell, Moritz Fleischmann, and Martin Savelsbergh. Time slot management in attended home delivery. Transportation Science, 45(3):435-449, 2011.

Enrico Angelelli, M. Grazia Speranza, and Martin W.P. Savelsbergh. Competitive analysis for dynamic multiperiod uncapacitated routing problems. Networks, 49(4):308-317, 2007a.

Enrico Angelelli, Martin W.P. Savelsbergh, and M. Grazia Speranza. Competitive analysis of a dispatch policy for a dynamic multi-period routing problem. Operations Research Letters, 35(6): $713-721,2007 \mathrm{~b}$.

Claudia Archetti, Dominique Feillet, and M Grazia Speranza. Complexity of routing problems with release dates. European Journal of Operational Research, 247(3):797-803, 2015a.

Claudia Archetti, Ola Jabali, and M Grazia Speranza. Multi-period vehicle routing problem with due dates. Computers \& Operations Research, 61:122-134, 2015b.

Claudia Archetti, Elena Fernández, and Diana L Huerta-Muñoz. The flexible periodic vehicle routing problem. Computers \&S Operations Research, 85:58-70, 2017.

Edward J Beltrami and Lawrence D Bodin. Networks and vehicle routing for municipal waste collection. Networks, 4(1):65-94, 1974.

Dimitri P Bertsekas. Dynamic programming and suboptimal control: A survey from adp to mpc. European Journal of Control, 11(4-5):310-334, 2005.

Leandro C Coelho, Jean-François Cordeau, and Gilbert Laporte. The inventory-routing problem with transshipment. Computers $\&$ Operations Research, 39(11):2537-2548, 2012.

Simon Emde and Nils Boysen. One-dimensional vehicle scheduling with a front-end depot and non-crossing constraints. OR spectrum, 36(2):381-400, 2014.

Güneş Erdoğan, Maria Battarra, and Gilbert Laporte. Scheduling twin robots on a line. Naval Research Logistics (NRL), 61(2):119-130, 2014. 
A. Estrada-Moreno, M. Savelsbergh, A. A. Juan, and J. Panadero. Biased-randomized iterated local search for a multiperiod vehicle routing problem with price discounts for delivery flexibility. International Transactions in Operational Research, 26(4):1293-1314, 2019.

Peter M Francis, Karen R Smilowitz, and Michal Tzur. The period vehicle routing problem and its extensions. In The vehicle routing problem: latest advances and new challenges, pages 73-102. Springer, 2008.

Mathias A Klapp, Alan L Erera, and Alejandro Toriello. The one-dimensional dynamic dispatch waves problem. Transportation Science, 52(2):402-415, 2016.

Yaxian Li, George Nemhauser, and Martin Savelsbergh. Pricing for production and delivery flexibility in single-item lot-sizing. Computers 83 Operations Research, 39(12):3408 - 3419, 2012.

Thomas L Morin. Computational advances in dynamic programming. In Dynamic programming and its applications, pages 53-90. Elsevier, 1978.

Magalie Mourgaya and François Vanderbeck. The periodic vehicle routing problem: classification and heuristic. RAIRO-Operations Research, 40(2):169-194, 2006.

Damián Reyes, Alan L Erera, and Martin WP Savelsbergh. Complexity of routing problems with release dates and deadlines. European Journal of Operational Research, 266(1):29-34, 2018.

Kalyan Talluri and Garrett van Ryzin. The Theory and Practice of Revenue Management. Springer US, 2004.

Marlin W Ulmer, Ninja Soeffker, and Dirk C Mattfeld. Value function approximation for dynamic multi-period vehicle routing. European Journal of Operational Research, 269(3):883-899, 2018.

Tong Wang and Beril L Toktay. Inventory management with advance demand information and flexible delivery. Management Science, 54(4):716-732, 2008.

Xinan Yang, Arne K Strauss, Christine SM Currie, and Richard Eglese. Choice-based demand management and vehicle routing in e-fulfillment. Transportation science, 50(2):473-488, 2014.

Baris Yildiz and Martin Savelsbergh. Provenly High Quality Solutions for Meal Delivery Routing Problems . Transportation Science (Forthcoming), 2019a.

Baris Yildiz and Martin Savelsbergh. Service and capacity planning in crowd-sourced delivery. Transportation Research Part C: Emerging Technologies, 100:177-199, 2019b.

Chuqian Zhang, Yat-wah Wan, Jiyin Liu, and Richard J Linn. Dynamic crane deployment in container storage yards. Transportation Research Part B: Methodological, 36(6):537-555, 2002. 


\section{Appendix. Detailed computational results}

Table 2 presents the results for the experiment in which we assess the quality of the solutions produced by the distance-based offer sequence. We provide the identifier of the offer sequence used (column labeled ID), either a random sequence (1 through 100) or the distance-based sequence (DB) and the total cost of the solution associated with the offer sequence. The distribu tion cost when all orders are served on the preferred are also provide (UB).

Table 2: Solutions with random customer sequences

\begin{tabular}{|c|c|c|c|c|c|c|c|c|c|c|c|c|c|c|c|c|c|c|c|c|}
\hline \multirow[b]{2}{*}{ ID } & \multicolumn{6}{|c|}{ Number of Customers $(n)$} & \multicolumn{7}{|c|}{ Number of Customers $(n)$} & \multirow[b]{2}{*}{ ID } & \multicolumn{5}{|c|}{ Number of Customers $(n)$} & \multirow[b]{2}{*}{15} \\
\hline & 10 & 11 & 12 & 13 & 14 & 15 & ID & 10 & 11 & 12 & 13 & 14 & 15 & & 10 & 11 & 12 & 13 & 14 & \\
\hline 1 & 4.36 & 5.08 & 5.22 & 5.77 & 5.79 & 6.00 & 35 & 4.31 & 5.04 & 5.20 & 5.80 & 5.88 & 6.02 & 69 & 4.48 & 5.17 & 5.17 & 5.81 & 5.90 & 6.01 \\
\hline 2 & 4.40 & 5.00 & 5.15 & 5.73 & 5.85 & 6.06 & 36 & 4.47 & 5.16 & 5.10 & 5.80 & 5.74 & 6.16 & 70 & 4.35 & 4.98 & 5.21 & 5.72 & 5.82 & 6.17 \\
\hline 3 & 4.42 & 5.00 & 5.24 & 5.80 & 5.69 & 5.99 & 37 & 4.37 & 5.13 & 5.05 & 5.82 & 5.82 & 6.04 & 71 & 4.48 & 5.13 & 5.21 & 5.69 & 5.85 & 5.91 \\
\hline 4 & 4.52 & 5.12 & 5.23 & 5.83 & 5.91 & 5.94 & 38 & 4.35 & 5.17 & 5.20 & 5.80 & 5.91 & 6.11 & 72 & 4.53 & 5.09 & 5.20 & 5.77 & 5.91 & \\
\hline 5 & 4.46 & 5.03 & 5.10 & 5.82 & 5.87 & 6.11 & 39 & 4.36 & 5.01 & 5.02 & 5.74 & 5.92 & 6.11 & 73 & 4.49 & 5.08 & 5.21 & 5.84 & 5.92 & 5.97 \\
\hline 6 & 4.47 & 5.19 & 5.23 & 5.70 & 5.80 & 6.00 & 40 & 4.51 & 5.02 & 5.07 & 5.89 & 5.85 & 6.15 & 74 & 4.36 & 5.14 & 5.06 & 5.84 & 5.80 & 5.93 \\
\hline 7 & 4.34 & 5.15 & 5.06 & 5.86 & 5.74 & 6.10 & 41 & 4.45 & 5.12 & 5.14 & 5.80 & 5.89 & 6.10 & 75 & 4.50 & 5.18 & 5.20 & 5.73 & 5.86 & 5.99 \\
\hline 8 & 4.42 & 5.17 & 5.19 & 5.72 & 5.90 & 6.08 & 42 & 4.46 & 5.14 & 5.20 & 5.75 & 5.76 & 6.16 & 76 & 4.36 & 4.97 & 5.08 & 5.83 & 5.69 & 6.05 \\
\hline 9 & 4.47 & 5.01 & 5.25 & 5.78 & 5.90 & 6.04 & 43 & 4.54 & 5.16 & 5.21 & 5.85 & 5.72 & 6.09 & 77 & 4.37 & 5.20 & 5.11 & 5.83 & 5.84 & 5.96 \\
\hline 10 & 4.45 & 5.11 & 5.20 & 5.76 & 5.89 & 5.94 & 44 & 4.43 & 4.99 & 5.02 & 5.91 & 5.77 & 6.16 & 78 & 4.48 & 5.16 & 5.25 & 5.83 & 5.70 & 6.13 \\
\hline 11 & 4.36 & 5.05 & 5.07 & 5.71 & 5.90 & 6.07 & 45 & 4.47 & 4.99 & 5.06 & 5.67 & 5.91 & 6.11 & 79 & 4.50 & 5.20 & 5.22 & 5.78 & 5.90 & 5.98 \\
\hline 12 & 4.47 & 5.14 & 5.25 & 5.63 & 5.94 & 5.99 & 46 & 4.48 & 5.14 & 5.16 & 5.84 & 5.94 & 5.98 & 80 & 4.54 & 5.14 & 5.13 & 5.68 & 5.79 & 5.94 \\
\hline 13 & 4.45 & 5.12 & 5.14 & 5.67 & 5.78 & 6.09 & 47 & 4.50 & 5.11 & 5.23 & 5.70 & 5.82 & 6.15 & 81 & 4.35 & 4.99 & 5.15 & 5.81 & 5.83 & 5.98 \\
\hline 14 & 4.51 & 5.17 & 5.14 & 5.76 & 5.71 & 5.94 & 48 & 4.35 & 5.19 & 5.22 & 5.79 & 5.84 & 6.05 & 82 & 4.52 & 5.03 & 5.15 & 5.73 & 5.79 & 6.16 \\
\hline 15 & 4.48 & 5.03 & 5.05 & 5.70 & 5.87 & 6.01 & 49 & 4.44 & 5.11 & 5.24 & 5.64 & 5.79 & 6.05 & 83 & 4.51 & 4.95 & 5.20 & 5.61 & 5.89 & 5.93 \\
\hline 16 & 4.47 & 5.03 & 5.10 & 5.84 & 5.87 & 6.10 & 50 & 4.46 & 5.07 & 5.20 & 5.76 & 5.85 & 6.08 & 84 & 4.49 & 5.20 & 5.23 & 5.82 & 5.82 & 6.03 \\
\hline 17 & 4.30 & 5.18 & 5.21 & 5.75 & 5.92 & 5.93 & 51 & 4.44 & 5.13 & 5.11 & 5.81 & 5.68 & 5.99 & 85 & 4.48 & 4.99 & 5.10 & 5.70 & 5.74 & 6.08 \\
\hline 18 & 4.49 & 5.10 & 5.06 & 5.74 & 5.92 & 6.12 & 52 & 4.50 & 5.15 & 5.09 & 5.74 & 5.81 & 5.96 & 86 & 4.33 & 5.15 & 5.15 & 5.85 & 5.65 & 6.02 \\
\hline 19 & 4.46 & 5.15 & 5.25 & 5.88 & 5.75 & 6.22 & 53 & 4.43 & 5.13 & 5.18 & 5.91 & 5.75 & 6.06 & 87 & 4.53 & 5.06 & 5.03 & 5.70 & 5.91 & 6.02 \\
\hline 20 & 4.51 & 5.15 & 5.25 & 5.73 & 5.79 & 5.90 & 54 & 4.49 & 5.16 & 5.24 & 5.84 & 5.90 & 6.01 & 88 & 4.34 & 5.15 & 5.14 & 5.83 & 5.84 & 6.01 \\
\hline 21 & 4.44 & 5.13 & 5.12 & 5.78 & 5.93 & 6.05 & 55 & 4.44 & 5.19 & 5.15 & 5.80 & 5.82 & 6.06 & 89 & 4.30 & 5.14 & 5.16 & 5.65 & 5.82 & \\
\hline 22 & 4.47 & 5.11 & 5.07 & 5.73 & 5.88 & 5.95 & 56 & 4.49 & 4.98 & 5.21 & 5.87 & 5.67 & 5.99 & 90 & 4.51 & 5.04 & 5.20 & 5.65 & 5.79 & 6.03 \\
\hline 23 & 4.49 & 5.22 & 5.24 & 5.84 & 5.78 & 5.97 & 57 & 4.49 & 5.08 & 5.16 & 5.78 & 5.86 & 6.11 & 91 & 4.48 & 5.10 & 5.15 & 5.68 & 5.85 & 5.97 \\
\hline 24 & 4.53 & 5.02 & 5.19 & 5.84 & 5.91 & 6.12 & 58 & 4.46 & 5.1 & 5.08 & 5.78 & 5.82 & 6.0 & 92 & 4.43 & 5.19 & 5.24 & 5.87 & 5.93 & 6. \\
\hline 25 & 4.32 & 4.99 & 5.22 & 5.80 & 5.89 & 6.02 & 59 & 4.33 & 5.09 & 5.24 & 5.75 & 5.92 & 6.0 & 93 & 4.48 & 5.13 & 5.08 & 5.63 & 5.91 & 5.98 \\
\hline 26 & 4.50 & 5.02 & 5.11 & 5.73 & 5.71 & 5.89 & 60 & 4.34 & 5.15 & 5.04 & 5.83 & 5.88 & 6.07 & 94 & 4.46 & 5.05 & 5.24 & 5.61 & 5.83 & 6.16 \\
\hline 27 & 4.51 & 5.18 & 5.18 & 5.82 & 5.91 & 6.15 & 61 & 4.50 & 5.00 & 5.07 & 5.65 & 5.72 & 6.02 & 95 & 4.33 & 5.16 & 5.17 & 5.74 & 5.89 & \\
\hline 28 & 4.34 & 5.14 & 5.18 & 5.73 & 5.81 & 6.10 & 62 & 4.40 & 5.14 & 5.23 & 5.75 & 5.86 & 6.10 & 96 & 4.35 & 5.13 & 5.24 & 5.83 & 5.89 & 6.11 \\
\hline 29 & 4.48 & 5.17 & 5.16 & 5.67 & 5.90 & 6.04 & 63 & 4.45 & 5.16 & 5.21 & 5.78 & 5.86 & 6.21 & 97 & 4.40 & 5.18 & 5.22 & 5.73 & 5.81 & 6.10 \\
\hline 30 & 4.47 & 5.06 & 5.16 & 5.84 & 5.78 & 6.1 & 64 & 4.48 & 4.97 & 5.19 & 5.88 & 5.93 & 6.0 & 98 & 4.41 & 5.18 & 5.10 & 5.78 & 5.90 & \\
\hline 31 & 4.46 & 5.12 & 5.16 & 5.89 & 5.95 & 5.96 & 65 & 4. & 5. & 5.14 & 5.75 & 5.84 & 6. & 99 & 4.43 & 5.08 & 5.05 & 5.81 & 5.85 & 6. \\
\hline 32 & 4.36 & 5.11 & 5.19 & 5.78 & 5.80 & 6.09 & 66 & 4.45 & 5.11 & 5.29 & 5.76 & 5.93 & 6.06 & 100 & 4.37 & 4.96 & 5.02 & 5.74 & 5.85 & \\
\hline 33 & 4.47 & 4.97 & 5.12 & 5.69 & 5.81 & 5.94 & 67 & 4.44 & 5.09 & 5.22 & 5.78 & 5.92 & 6.12 & FCF & 4.34 & 4.97 & 5.03 & 5.65 & 5.70 & 5. \\
\hline 34 & 4.49 & 5.11 & 5.05 & 5.66 & 5.77 & 6.14 & 68 & 4.48 & 5.11 & 5.07 & 5.74 & 5.92 & 6.12 & UB & 6.95 & 8.20 & 8.20 & 8.90 & 8.90 & 9.14 \\
\hline
\end{tabular}


Table 3 presents the results for E1 experiments. The columns labeled $n$ indicate the number of customers, seed shows the integer used as the random number generator seed, UB presents the distribution cost without discount offers, GS indicates the expected total cost (delivery and discount costs together) of the greedy heuristic solutions for the respective $\alpha$ values and PDFP indicates the expected cost of the offer policy suggested by DP-E.

Table 3: Solution results for the E1 problem instances

\begin{tabular}{|c|c|c|c|c|c|c|c|c|c|c|}
\hline \multirow[b]{2}{*}{$n$} & \multirow[b]{2}{*}{ seed } & \multirow[b]{2}{*}{ UB } & \multicolumn{2}{|c|}{$\alpha=0.05$} & \multicolumn{2}{|c|}{$\alpha=0.10$} & \multicolumn{2}{|c|}{$\alpha=0.20$} & \multicolumn{2}{|c|}{ DP-E } \\
\hline & & & GS & $\mathrm{DC}$ & GS & $\mathrm{DC}$ & GS & $\mathrm{DC}$ & PDFP & $\mathrm{DC}$ \\
\hline \multirow[t]{5}{*}{15} & 0 & 7.59 & 5.49 & 0.24 & 5.75 & 0.35 & 5.80 & 0.59 & 4.23 & 1.41 \\
\hline & 1 & 9.14 & 7.80 & 0.33 & 7.65 & 0.56 & 7.34 & 1.45 & 5.90 & 1.30 \\
\hline & 2 & 7.74 & 6.61 & 0.16 & 6.43 & 0.42 & 6.71 & 0.99 & 5.64 & 1.42 \\
\hline & 3 & 8.29 & 7.00 & 0.21 & 6.53 & 0.57 & 6.48 & 1.33 & 5.30 & 1.41 \\
\hline & 4 & 7.23 & 6.81 & 0.06 & 6.77 & 0.20 & 6.95 & 0.59 & 4.82 & 1.34 \\
\hline \multirow[t]{5}{*}{20} & 0 & 8.91 & 7.10 & 0.15 & 7.22 & 0.32 & 7.20 & 0.72 & 5.75 & 1.75 \\
\hline & 1 & 9.18 & 8.54 & 0.13 & 8.63 & 0.23 & 8.93 & 0.35 & 7.06 & 1.64 \\
\hline & 2 & 7.74 & 7.74 & 0.00 & 7.74 & 0.00 & 7.74 & 0.00 & 5.91 & 1.50 \\
\hline & 3 & 8.40 & 7.15 & 0.22 & 7.13 & 0.53 & 7.61 & 1.20 & 5.75 & 1.37 \\
\hline & 4 & 7.46 & 6.52 & 0.17 & 6.97 & 0.26 & 7.15 & 0.59 & 5.37 & 1.59 \\
\hline \multirow[t]{5}{*}{25} & 0 & 9.35 & 7.38 & 0.28 & 8.98 & 0.12 & 8.97 & 0.34 & 6.77 & 1.90 \\
\hline & 1 & 9.18 & 8.67 & 0.12 & 8.80 & 0.16 & 8.93 & 0.35 & 7.75 & 1.39 \\
\hline & 2 & 7.74 & 7.74 & 0.00 & 7.74 & 0.00 & 7.74 & 0.00 & 6.00 & 1.51 \\
\hline & 3 & 8.56 & 7.36 & 0.24 & 7.40 & 0.59 & 7.92 & 1.44 & 6.19 & 1.33 \\
\hline & 4 & 8.25 & 7.04 & 0.25 & 7.39 & 0.51 & 7.76 & 0.82 & 6.31 & 1.44 \\
\hline \multirow[t]{5}{*}{30} & 0 & 9.51 & 8.33 & 0.15 & 9.51 & 0.00 & 9.51 & 0.00 & 7.58 & 1.57 \\
\hline & 1 & 9.73 & 9.24 & 0.21 & 9.30 & 0.38 & 9.73 & 0.00 & 8.33 & 1.39 \\
\hline & 2 & 7.74 & 7.74 & 0.00 & 7.74 & 0.00 & 7.74 & 0.00 & 6.18 & 1.50 \\
\hline & 3 & 8.70 & 7.69 & 0.17 & 7.51 & 0.37 & 7.73 & 0.83 & 6.62 & 1.26 \\
\hline & 4 & 8.96 & 8.03 & 0.24 & 8.20 & 0.45 & 8.39 & 0.62 & 7.10 & 1.40 \\
\hline \multirow[t]{5}{*}{35} & 0 & 9.51 & 8.73 & 0.18 & 9.51 & 0.00 & 9.51 & 0.00 & 8.29 & 1.27 \\
\hline & 1 & 9.73 & 9.25 & 0.21 & 9.30 & 0.38 & 9.73 & 0.00 & 8.39 & 1.35 \\
\hline & 2 & 8.87 & 8.40 & 0.05 & 8.37 & 0.12 & 8.42 & 0.29 & 7.36 & 1.55 \\
\hline & 3 & 9.35 & 8.11 & 0.42 & 7.91 & 0.35 & 8.99 & 0.81 & 7.11 & 1.34 \\
\hline & 4 & 8.96 & 8.49 & 0.17 & 8.22 & 0.46 & 8.96 & 0.00 & 7.43 & 1.34 \\
\hline \multirow[t]{5}{*}{40} & 0 & 9.60 & 8.81 & 0.18 & 9.60 & 0.00 & 9.60 & 0.00 & 8.46 & 1.15 \\
\hline & 1 & 9.73 & 9.36 & 0.20 & 9.45 & 0.44 & 9.73 & 0.00 & 8.76 & 1.09 \\
\hline & 2 & 8.98 & 8.98 & 0.00 & 8.98 & 0.00 & 8.98 & 0.00 & 7.88 & 1.42 \\
\hline & 3 & 9.35 & 8.45 & 0.39 & 8.70 & 0.26 & 8.99 & 0.81 & 7.76 & 1.12 \\
\hline & 4 & 8.96 & 8.53 & 0.17 & 8.22 & 0.46 & 8.96 & 0.00 & 7.64 & 1.07 \\
\hline
\end{tabular}


Table 4 presents the results for E2 experiments. As described in Section 4.1, the columns "Inverse Triangular", "Triangular" and "Uniform" indicate the temporal distributions of the customers in the respective instance. The other columns present the same information as in Table 3.

Table 4: Solution results for the E2 problem instances

\begin{tabular}{|c|c|c|c|c|c|c|c|c|c|c|}
\hline \multirow[b]{2}{*}{$n$} & \multirow[b]{2}{*}{ seed } & \multicolumn{3}{|c|}{ Inverse Triangular } & \multicolumn{3}{|c|}{ Triangular } & \multicolumn{3}{|c|}{ Uniform } \\
\hline & & UB & PDFP & $\mathrm{DC}$ & $\mathrm{UB}$ & PDFP & $\mathrm{DC}$ & UB & PDFP & $\mathrm{DC}$ \\
\hline \multirow[t]{5}{*}{15} & 0 & 6.43 & 4.87 & 0.85 & 8.47 & 5.13 & 1.77 & 7.59 & 4.23 & 1.41 \\
\hline & 1 & 8.59 & 6.06 & 1.24 & 5.01 & 4.25 & 0.81 & 9.14 & 5.90 & 1.30 \\
\hline & 2 & 6.74 & 4.92 & 0.64 & 7.84 & 5.30 & 0.89 & 7.74 & 5.64 & 1.42 \\
\hline & 3 & 5.51 & 4.48 & 0.58 & 5.36 & 3.61 & 0.91 & 8.29 & 5.30 & 1.41 \\
\hline & 4 & 8.52 & 6.15 & 1.48 & 6.39 & 4.13 & 1.34 & 7.23 & 4.82 & 1.34 \\
\hline \multirow[t]{5}{*}{20} & 0 & 6.81 & 5.60 & 0.98 & 8.48 & 5.39 & 1.96 & 8.91 & 5.75 & 1.75 \\
\hline & 1 & 8.93 & 6.75 & 1.25 & 6.35 & 5.22 & 0.93 & 9.18 & 7.06 & 1.64 \\
\hline & 2 & 7.92 & 5.81 & 0.94 & 8.53 & 5.73 & 1.46 & 7.74 & 5.91 & 1.50 \\
\hline & 3 & 8.62 & 6.08 & 1.04 & 7.43 & 5.17 & 1.29 & 8.40 & 5.75 & 1.37 \\
\hline & 4 & 8.59 & 6.46 & 1.25 & 6.39 & 4.39 & 1.28 & 7.46 & 5.37 & 1.59 \\
\hline \multirow[t]{5}{*}{25} & 0 & 8.11 & 6.28 & 1.21 & 8.48 & 5.61 & 1.89 & 9.35 & 6.77 & 1.90 \\
\hline & 1 & 9.00 & 7.34 & 1.33 & 6.35 & 5.25 & 0.76 & 9.18 & 7.75 & 1.39 \\
\hline & 2 & 8.45 & 6.93 & 1.25 & 8.60 & 6.00 & 1.10 & 7.74 & 6.00 & 1.51 \\
\hline & 3 & 8.62 & 6.14 & 1.08 & 7.80 & 5.36 & 1.27 & 8.56 & 6.19 & 1.33 \\
\hline & 4 & 8.66 & 6.74 & 1.30 & 6.61 & 4.90 & 1.10 & 8.25 & 6.31 & 1.44 \\
\hline \multirow[t]{5}{*}{30} & 0 & 8.11 & 6.65 & 1.23 & 8.48 & 6.35 & 1.64 & 9.51 & 7.58 & 1.57 \\
\hline & 1 & 9.00 & 7.45 & 1.30 & 6.44 & 5.54 & 0.67 & 9.73 & 8.33 & 1.39 \\
\hline & 2 & 8.47 & 7.59 & 0.93 & 8.60 & 6.13 & 1.17 & 7.74 & 6.18 & 1.50 \\
\hline & 3 & 9.21 & 7.00 & 1.36 & 7.80 & 5.88 & 1.40 & 8.70 & 6.62 & 1.26 \\
\hline & 4 & 8.66 & 7.26 & 1.16 & 6.96 & 5.16 & 1.23 & 8.96 & 7.10 & 1.40 \\
\hline \multirow[t]{5}{*}{35} & 0 & 8.11 & 7.06 & 1.25 & 8.48 & 6.48 & 1.51 & 9.51 & 8.29 & 1.27 \\
\hline & 1 & 9.00 & 7.80 & 1.20 & 6.44 & 5.55 & 0.65 & 9.73 & 8.39 & 1.35 \\
\hline & 2 & 8.98 & 8.12 & 0.89 & 8.60 & 6.32 & 1.17 & 8.87 & 7.36 & 1.55 \\
\hline & 3 & 9.22 & 7.82 & 1.21 & 7.86 & 6.29 & 1.33 & 9.35 & 7.11 & 1.34 \\
\hline & 4 & 8.95 & 7.87 & 0.98 & 7.08 & 5.76 & 1.17 & 8.96 & 7.43 & 1.34 \\
\hline \multirow[t]{5}{*}{40} & 0 & 8.40 & 7.66 & 1.00 & 8.48 & 6.57 & 1.33 & 9.60 & 8.46 & 1.15 \\
\hline & 1 & 9.00 & 8.08 & 1.17 & 7.99 & 5.97 & 0.55 & 9.73 & 8.76 & 1.09 \\
\hline & 2 & 8.98 & 8.42 & 0.70 & 8.60 & 6.54 & 1.20 & 8.98 & 7.88 & 1.42 \\
\hline & 3 & 9.22 & 8.24 & 1.10 & 8.06 & 6.61 & 1.18 & 9.35 & 7.76 & 1.12 \\
\hline & 4 & 8.95 & 7.87 & 0.98 & 7.08 & 5.77 & 1.16 & 8.96 & 7.64 & 1.07 \\
\hline
\end{tabular}


Table 5 presents the results for E3 experiments. As described in Section 4.1, the columns $f_{1}, f_{2}$ and $f_{3}$ indicate the spatial distributions of the customer in the respective problem instances. The other columns present the same information as in Table 3.

Table 5: Solution results for the E3 problem instances

\begin{tabular}{|c|c|c|c|c|c|c|c|c|c|c|}
\hline \multirow[b]{2}{*}{$n$} & \multirow[b]{2}{*}{ seed } & \multicolumn{3}{|c|}{$\overline{f_{1}}$} & \multicolumn{3}{|c|}{$f_{2}$} & \multicolumn{3}{|c|}{$f_{3}$} \\
\hline & & UB & PDFP & $\mathrm{DC}$ & UB & PDFP & $\mathrm{DC}$ & UB & PDFP & $\mathrm{DC}$ \\
\hline \multirow[t]{5}{*}{15} & 0 & 5.53 & 3.18 & 0.93 & 7.59 & 4.23 & 1.41 & 8.63 & 5.00 & 1.77 \\
\hline & 1 & 7.25 & 4.58 & 1.08 & 9.14 & 5.90 & 1.30 & 9.55 & 6.52 & 1.57 \\
\hline & 2 & 5.90 & 4.25 & 1.03 & 7.74 & 5.64 & 1.42 & 8.68 & 6.56 & 1.64 \\
\hline & 3 & 6.52 & 4.27 & 1.02 & 8.29 & 5.30 & 1.41 & 9.04 & 6.30 & 1.59 \\
\hline & 4 & 4.93 & 3.31 & 0.86 & 7.23 & 4.82 & 1.34 & 8.46 & 5.88 & 1.68 \\
\hline \multirow[t]{5}{*}{20} & 0 & 7.08 & 4.47 & 1.32 & 8.91 & 5.75 & 1.75 & 9.43 & 6.51 & 1.79 \\
\hline & 1 & 7.38 & 5.49 & 1.25 & 9.18 & 7.06 & 1.64 & 9.58 & 7.82 & 1.78 \\
\hline & 2 & 5.90 & 4.40 & 1.08 & 7.74 & 5.91 & 1.50 & 8.68 & 7.02 & 1.73 \\
\hline & 3 & 6.60 & 4.54 & 1.01 & 8.40 & 5.75 & 1.37 & 9.12 & 6.98 & 1.48 \\
\hline & 4 & 5.17 & 3.70 & 1.05 & 7.46 & 5.37 & 1.59 & 8.59 & 6.51 & 1.89 \\
\hline \multirow[t]{5}{*}{25} & 0 & 7.85 & 5.36 & 1.56 & 9.35 & 6.77 & 1.90 & 9.66 & 7.43 & 1.78 \\
\hline & 1 & 7.38 & 5.99 & 1.05 & 9.18 & 7.75 & 1.39 & 9.58 & 8.46 & 1.48 \\
\hline & 2 & 5.90 & 4.47 & 1.09 & 7.74 & 6.00 & 1.51 & 8.68 & 7.11 & 1.70 \\
\hline & 3 & 6.82 & 4.84 & 1.01 & 8.56 & 6.19 & 1.33 & 9.21 & 7.52 & 1.28 \\
\hline & 4 & 5.89 & 4.40 & 0.96 & 8.25 & 6.31 & 1.44 & 9.07 & 7.41 & 1.65 \\
\hline \multirow[t]{5}{*}{30} & 0 & 8.04 & 6.06 & 1.41 & 9.51 & 7.58 & 1.57 & 9.75 & 8.13 & 1.31 \\
\hline & 1 & 8.44 & 6.68 & 1.30 & 9.73 & 8.33 & 1.39 & 9.86 & 8.83 & 1.40 \\
\hline & 2 & 5.90 & 4.58 & 1.11 & 7.74 & 6.18 & 1.50 & 8.68 & 7.43 & 1.58 \\
\hline & 3 & 7.08 & 5.23 & 1.05 & 8.70 & 6.62 & 1.26 & 9.28 & 7.83 & 1.11 \\
\hline & 4 & 6.97 & 5.29 & 1.03 & 8.96 & 7.10 & 1.40 & 9.46 & 8.10 & 1.47 \\
\hline \multirow[t]{5}{*}{35} & 0 & 8.04 & 6.56 & 1.25 & 9.51 & 8.29 & 1.27 & 9.75 & 8.90 & 0.98 \\
\hline & 1 & 8.44 & 6.71 & 1.29 & 9.73 & 8.39 & 1.35 & 9.86 & 9.01 & 1.21 \\
\hline & 2 & 6.92 & 5.53 & 1.25 & 8.87 & 7.36 & 1.55 & 9.41 & 8.45 & 1.31 \\
\hline & 3 & 7.83 & 5.64 & 1.16 & 9.35 & 7.11 & 1.34 & 9.66 & 8.26 & 1.05 \\
\hline & 4 & 6.97 & 5.51 & 1.01 & 8.96 & 7.43 & 1.34 & 9.46 & 8.44 & 1.30 \\
\hline \multirow[t]{5}{*}{40} & 0 & 8.17 & 6.75 & 1.17 & 9.60 & 8.46 & 1.15 & 9.80 & 8.99 & 0.88 \\
\hline & 1 & 8.44 & 7.03 & 1.11 & 9.73 & 8.76 & 1.09 & 9.86 & 9.25 & 1.07 \\
\hline & 2 & 7.14 & 6.02 & 1.22 & 8.98 & 7.88 & 1.42 & 9.47 & 8.82 & 1.07 \\
\hline & 3 & 7.83 & 6.15 & 1.02 & 9.35 & 7.76 & 1.12 & 9.66 & 8.69 & 0.84 \\
\hline & 4 & 6.97 & 5.64 & 0.85 & 8.96 & 7.64 & 1.07 & 9.46 & 8.67 & 0.90 \\
\hline
\end{tabular}


Table 6 presents the results for E4 experiments. As described in Section 4.1, the columns $k=0.125, k=0.25, k=0.5$ and $T P$ (threshold probability) customer offer acceptance probability families for the respective problem instances. The other columns present the same information as in Table 3.

Table 6: Solution results for the E4 problem instances

\begin{tabular}{|c|c|c|c|c|c|c|c|c|c|c|}
\hline \multirow[b]{2}{*}{$n$} & \multirow[b]{2}{*}{ seed } & \multirow[b]{2}{*}{ UB } & \multicolumn{2}{|c|}{$k=0.125$} & \multicolumn{2}{|c|}{$k=0.25$} & \multicolumn{2}{|c|}{$k=0.5$} & \multicolumn{2}{|c|}{$\mathrm{TP}$} \\
\hline & & & PDFP & $\mathrm{DC}$ & PDFP & $\mathrm{DC}$ & PDFP & $\mathrm{DC}$ & PDFP & $\mathrm{DC}$ \\
\hline \multirow[t]{5}{*}{15} & 0 & 5.53 & 3.18 & 0.93 & 4.23 & 1.41 & 5.00 & 1.77 & 3.53 & 1.57 \\
\hline & 1 & 7.25 & 4.58 & 1.08 & 5.90 & 1.30 & 6.52 & 1.57 & 5.70 & 1.88 \\
\hline & 2 & 5.90 & 4.25 & 1.03 & 5.64 & 1.42 & 6.56 & 1.64 & 5.47 & 2.03 \\
\hline & 3 & 6.52 & 4.27 & 1.02 & 5.30 & 1.41 & 6.30 & 1.59 & 4.99 & 2.48 \\
\hline & 4 & 4.93 & 3.31 & 0.86 & 4.82 & 1.34 & 5.88 & 1.68 & 4.11 & 1.30 \\
\hline \multirow[t]{5}{*}{20} & 0 & 7.08 & 4.47 & 1.32 & 5.75 & 1.75 & 6.51 & 1.79 & 4.99 & 1.04 \\
\hline & 1 & 7.38 & 5.49 & 1.25 & 7.06 & 1.64 & 7.82 & 1.78 & 6.85 & 2.94 \\
\hline & 2 & 5.90 & 4.40 & 1.08 & 5.91 & 1.50 & 7.02 & 1.73 & 5.59 & 2.15 \\
\hline & 3 & 6.60 & 4.54 & 1.01 & 5.75 & 1.37 & 6.98 & 1.48 & 5.75 & 3.18 \\
\hline & 4 & 5.17 & 3.70 & 1.05 & 5.37 & 1.59 & 6.51 & 1.89 & 4.47 & 1.66 \\
\hline \multirow[t]{5}{*}{25} & 0 & 7.85 & 5.36 & 1.56 & 6.77 & 1.90 & 7.43 & 1.78 & 5.79 & 1.85 \\
\hline & 1 & 7.38 & 5.99 & 1.05 & 7.75 & 1.39 & 8.46 & 1.48 & 7.41 & 1.78 \\
\hline & 2 & 5.90 & 4.47 & 1.09 & 6.00 & 1.51 & 7.11 & 1.70 & 5.59 & 2.15 \\
\hline & 3 & 6.82 & 4.84 & 1.01 & 6.19 & 1.33 & 7.52 & 1.28 & 6.34 & 3.77 \\
\hline & 4 & 5.89 & 4.40 & 0.96 & 6.31 & 1.44 & 7.41 & 1.65 & 5.29 & 1.84 \\
\hline \multirow[t]{5}{*}{30} & 0 & 8.04 & 6.06 & 1.41 & 7.58 & 1.57 & 8.13 & 1.31 & 7.03 & 3.34 \\
\hline & 1 & 8.44 & 6.68 & 1.30 & 8.33 & 1.39 & 8.83 & 1.40 & 7.69 & 1.78 \\
\hline & 2 & 5.90 & 4.58 & 1.11 & 6.18 & 1.50 & 7.43 & 1.58 & 5.72 & 2.29 \\
\hline & 3 & 7.08 & 5.23 & 1.05 & 6.62 & 1.26 & 7.83 & 1.11 & 6.83 & 2.48 \\
\hline & 4 & 6.97 & 5.29 & 1.03 & 7.10 & 1.40 & 8.10 & 1.47 & 6.77 & 3.20 \\
\hline \multirow[t]{5}{*}{35} & 0 & 8.04 & 6.56 & 1.25 & 8.29 & 1.27 & 8.90 & 0.98 & 7.64 & 2.01 \\
\hline & 1 & 8.44 & 6.71 & 1.29 & 8.39 & 1.35 & 9.01 & 1.21 & 7.83 & 1.93 \\
\hline & 2 & 6.92 & 5.53 & 1.25 & 7.36 & 1.55 & 8.45 & 1.31 & 7.25 & 3.82 \\
\hline & 3 & 7.83 & 5.64 & 1.16 & 7.11 & 1.34 & 8.26 & 1.05 & 7.43 & 1.19 \\
\hline & 4 & 6.97 & 5.51 & 1.01 & 7.43 & 1.34 & 8.44 & 1.30 & 6.89 & 3.32 \\
\hline \multirow[t]{5}{*}{40} & 0 & 8.17 & 6.75 & 1.17 & 8.46 & 1.15 & 8.99 & 0.88 & 7.73 & 2.01 \\
\hline & 1 & 8.44 & 7.03 & 1.11 & 8.76 & 1.09 & 9.25 & 1.07 & 7.97 & 2.06 \\
\hline & 2 & 7.14 & 6.02 & 1.22 & 7.88 & 1.42 & 8.82 & 1.07 & 7.72 & 2.37 \\
\hline & 3 & 7.83 & 6.15 & 1.02 & 7.76 & 1.12 & 8.69 & 0.84 & 7.82 & 2.15 \\
\hline & 4 & 6.97 & 5.64 & 0.85 & 7.64 & 1.07 & 8.67 & 0.90 & 7.23 & 1.78 \\
\hline
\end{tabular}


Table 7 presents the results for E5 experiments. As described in Section 4.1, the columns $r=0.9, r=1, r=1.1, r=1.2$ and $r=1.3$ indicate the revenue multipliers used to generate respective problem instances. The other columns present the same information as in Table 3.

Table 7: Solution results for the E5 problem instances

\begin{tabular}{|c|c|c|c|c|c|c|c|c|c|c|c|c|}
\hline \multirow[b]{2}{*}{$n$} & \multirow[b]{2}{*}{ seed } & \multirow[b]{2}{*}{ UB } & \multicolumn{2}{|c|}{$r=0.9$} & \multicolumn{2}{|c|}{$r=1$} & \multicolumn{2}{|c|}{$r=1.1$} & \multicolumn{2}{|c|}{$r=1.2$} & \multicolumn{2}{|c|}{$r=1.3$} \\
\hline & & & PDFP & $\mathrm{DC}$ & PDFP & $\mathrm{DC}$ & PDFP & $\mathrm{DC}$ & PDFP & $\mathrm{DC}$ & PDFP & $\mathrm{DC}$ \\
\hline \multirow[t]{5}{*}{15} & 0 & 7.59 & 3.99 & 1.35 & 4.12 & 1.38 & 4.23 & 1.41 & 4.34 & 1.41 & 4.44 & 1.39 \\
\hline & 1 & 9.14 & 5.69 & 1.23 & 5.80 & 1.27 & 5.90 & 1.30 & 5.99 & 1.31 & 6.07 & 1.32 \\
\hline & 2 & 7.74 & 5.43 & 1.48 & 5.54 & 1.45 & 5.64 & 1.42 & 5.73 & 1.40 & 5.80 & 1.37 \\
\hline & 3 & 8.29 & 5.08 & 1.45 & 5.19 & 1.43 & 5.30 & 1.41 & 5.38 & 1.40 & 5.46 & 1.38 \\
\hline & 4 & 7.23 & 4.59 & 1.32 & 4.71 & 1.34 & 4.82 & 1.34 & 4.91 & 1.34 & 5.00 & 1.34 \\
\hline \multirow[t]{5}{*}{20} & 0 & 8.91 & 5.45 & 1.79 & 5.61 & 1.77 & 5.75 & 1.75 & 5.87 & 1.69 & 5.98 & 1.64 \\
\hline & 1 & 9.18 & 6.80 & 1.75 & 6.94 & 1.69 & 7.06 & 1.64 & 7.16 & 1.60 & 7.25 & 1.55 \\
\hline & 2 & 7.74 & 5.68 & 1.59 & 5.80 & 1.55 & 5.91 & 1.50 & 6.00 & 1.47 & 6.08 & 1.42 \\
\hline & 3 & 8.40 & 5.54 & 1.51 & 5.66 & 1.40 & 5.75 & 1.37 & 5.84 & 1.35 & 5.91 & 1.33 \\
\hline & 4 & 7.46 & 5.09 & 1.63 & 5.24 & 1.62 & 5.37 & 1.59 & 5.48 & 1.55 & 5.58 & 1.51 \\
\hline \multirow[t]{5}{*}{25} & 0 & 9.35 & 6.45 & 1.99 & 6.62 & 1.94 & 6.77 & 1.90 & 6.90 & 1.83 & 7.02 & 1.75 \\
\hline & 1 & 9.18 & 7.53 & 1.56 & 7.65 & 1.47 & 7.75 & 1.39 & 7.84 & 1.32 & 7.91 & 1.26 \\
\hline & 2 & 7.74 & 5.77 & 1.62 & 5.89 & 1.56 & 6.00 & 1.51 & 6.10 & 1.47 & 6.18 & 1.42 \\
\hline & 3 & 8.56 & 5.99 & 1.42 & 6.10 & 1.37 & 6.19 & 1.33 & 6.26 & 1.30 & 6.33 & 1.27 \\
\hline & 4 & 8.25 & 6.07 & 1.54 & 6.20 & 1.49 & 6.31 & 1.44 & 6.41 & 1.39 & 6.49 & 1.31 \\
\hline \multirow[t]{5}{*}{30} & 0 & 9.51 & 7.32 & 1.69 & 7.46 & 1.63 & 7.58 & 1.57 & 7.69 & 1.50 & 7.78 & 1.42 \\
\hline & 1 & 9.73 & 8.11 & 1.55 & 8.23 & 1.46 & 8.33 & 1.39 & 8.41 & 1.32 & 8.49 & 1.26 \\
\hline & 2 & 7.74 & 5.94 & 1.65 & 6.07 & 1.57 & 6.18 & 1.50 & 6.27 & 1.44 & 6.35 & 1.38 \\
\hline & 3 & 8.70 & 6.43 & 1.38 & 6.53 & 1.31 & 6.62 & 1.26 & 6.69 & 1.22 & 6.75 & 1.18 \\
\hline & 4 & 8.96 & 6.88 & 1.53 & 7.00 & 1.46 & 7.10 & 1.40 & 7.19 & 1.35 & 7.26 & 1.31 \\
\hline \multirow[t]{5}{*}{35} & 0 & 9.51 & 8.09 & 1.54 & 8.20 & 1.38 & 8.29 & 1.27 & 8.37 & 1.18 & 8.43 & 1.11 \\
\hline & 1 & 9.73 & 8.17 & 1.52 & 8.29 & 1.43 & 8.39 & 1.35 & 8.47 & 1.28 & 8.54 & 1.22 \\
\hline & 2 & 8.87 & 7.13 & 1.79 & 7.26 & 1.66 & 7.36 & 1.55 & 7.45 & 1.46 & 7.52 & 1.38 \\
\hline & 3 & 9.35 & 6.91 & 1.47 & 7.02 & 1.40 & 7.11 & 1.34 & 7.18 & 1.29 & 7.25 & 1.25 \\
\hline & 4 & 8.96 & 7.21 & 1.52 & 7.33 & 1.42 & 7.43 & 1.34 & 7.51 & 1.27 & 7.57 & 1.20 \\
\hline \multirow[t]{5}{*}{40} & 0 & 9.60 & 8.27 & 1.39 & 8.38 & 1.25 & 8.46 & 1.15 & 8.53 & 1.07 & 8.59 & 1.01 \\
\hline & 1 & 9.73 & 8.57 & 1.42 & 8.68 & 1.29 & 8.76 & 1.09 & 8.83 & 1.02 & 8.88 & 0.95 \\
\hline & 2 & 8.98 & 7.67 & 1.68 & 7.79 & 1.53 & 7.88 & 1.42 & 7.96 & 1.33 & 8.03 & 1.25 \\
\hline & 3 & 9.35 & 7.60 & 1.25 & 7.69 & 1.18 & 7.76 & 1.12 & 7.82 & 1.07 & 7.87 & 1.03 \\
\hline & 4 & 8.96 & 7.48 & 1.21 & 7.57 & 1.13 & 7.64 & 1.07 & 7.70 & 1.02 & 7.75 & 0.98 \\
\hline
\end{tabular}


Table 8 presents the results for E6 experiments. As described in Section 4.1, the columns $v=1.25, v=1.5, v=2$ and $\mathrm{UC}$ (uncapacitated) indicate the vehicle capacity levels for the respective problem instances. The other columns present the same information as in Table 3.

Table 8: Solution results for the E6 problem instances

\begin{tabular}{|c|c|c|c|c|c|c|c|c|c|c|c|c|c|}
\hline \multirow[b]{2}{*}{$n$} & \multirow[b]{2}{*}{ seed } & \multicolumn{3}{|c|}{$v=1.25$} & \multicolumn{3}{|c|}{$v=1.5$} & \multicolumn{3}{|c|}{$v=2$} & \multicolumn{3}{|c|}{$\mathrm{UC}$} \\
\hline & & UB & PDFP & $\mathrm{DC}$ & UB & PDFP & $\mathrm{DC}$ & UB & PDFP & $\mathrm{DC}$ & UB & PDFP & $\mathrm{DC}$ \\
\hline \multirow[t]{5}{*}{15} & 0 & 10.19 & 8.09 & 0.86 & 9.28 & 6.93 & 0.85 & 7.75 & 5.80 & 0.74 & 7.59 & 4.23 & 1.41 \\
\hline & 1 & 10.34 & 7.73 & 0.55 & 9.14 & 6.81 & 0.82 & 9.14 & 6.20 & 1.08 & 9.14 & 5.90 & 1.30 \\
\hline & 2 & 7.98 & 7.38 & 0.34 & 7.74 & 6.72 & 0.68 & 7.74 & 6.14 & 0.97 & 7.74 & 5.64 & 1.42 \\
\hline & 3 & 8.56 & 7.38 & 0.67 & 8.29 & 6.44 & 0.71 & 8.29 & 5.86 & 1.00 & 8.29 & 5.30 & 1.41 \\
\hline & 4 & 7.23 & 6.45 & 0.43 & 7.23 & 5.95 & 0.60 & 7.23 & 5.31 & 0.86 & 7.23 & 4.82 & 1.34 \\
\hline \multirow[t]{5}{*}{20} & 0 & 10.60 & 8.23 & 0.77 & 9.39 & 7.43 & 0.60 & 8.91 & 6.61 & 1.00 & 8.91 & 5.75 & 1.75 \\
\hline & 1 & 10.38 & 8.24 & 0.61 & 9.18 & 7.82 & 0.71 & 9.18 & 7.31 & 1.22 & 9.18 & 7.06 & 1.64 \\
\hline & 2 & 7.74 & 6.92 & 0.55 & 7.74 & 6.58 & 0.87 & 7.74 & 6.26 & 1.04 & 7.74 & 5.91 & 1.50 \\
\hline & 3 & 8.68 & 6.84 & 0.87 & 8.40 & 6.22 & 0.94 & 8.40 & 5.92 & 1.12 & 8.40 & 5.75 & 1.37 \\
\hline & 4 & 7.46 & 6.56 & 0.43 & 7.46 & 6.22 & 0.54 & 7.46 & 5.75 & 1.10 & 7.46 & 5.37 & 1.59 \\
\hline \multirow[t]{5}{*}{25} & 0 & 11.03 & 9.12 & 0.64 & 9.51 & 8.30 & 0.96 & 9.35 & 7.53 & 1.23 & 9.35 & 6.77 & 1.90 \\
\hline & 1 & 9.54 & 8.89 & 0.48 & 9.18 & 8.31 & 0.73 & 9.18 & 7.98 & 0.95 & 9.18 & 7.75 & 1.39 \\
\hline & 2 & 7.93 & 7.26 & 0.54 & 7.80 & 6.84 & 0.75 & 7.74 & 6.50 & 0.95 & 7.74 & 6.00 & 1.51 \\
\hline & 3 & 8.56 & 7.15 & 0.60 & 8.56 & 6.80 & 0.70 & 8.56 & 6.36 & 1.04 & 8.56 & 6.19 & 1.33 \\
\hline & 4 & 10.33 & 8.19 & 0.64 & 8.79 & 7.40 & 0.71 & 8.25 & 6.64 & 1.03 & 8.25 & 6.31 & 1.44 \\
\hline \multirow[t]{5}{*}{30} & 0 & 9.67 & 8.86 & 0.83 & 9.51 & 8.23 & 0.91 & 9.51 & 7.84 & 1.15 & 9.51 & 7.58 & 1.57 \\
\hline & 1 & 10.44 & 9.59 & 0.69 & 10.09 & 9.08 & 0.81 & 9.73 & 8.47 & 1.10 & 9.73 & 8.33 & 1.39 \\
\hline & 2 & 7.93 & 7.31 & 0.56 & 7.80 & 6.97 & 0.71 & 7.74 & 6.60 & 0.90 & 7.74 & 6.18 & 1.50 \\
\hline & 3 & 8.71 & 7.07 & 0.70 & 8.70 & 6.88 & 0.89 & 8.70 & 6.69 & 1.09 & 8.70 & 6.62 & 1.26 \\
\hline & 4 & 11.70 & 9.04 & 0.77 & 10.16 & 8.30 & 0.88 & 8.96 & 7.47 & 1.04 & 8.96 & 7.10 & 1.40 \\
\hline \multirow[t]{5}{*}{35} & 0 & 9.98 & 9.38 & 0.33 & 9.51 & 8.85 & 0.75 & 9.51 & 8.45 & 0.93 & 9.51 & 8.29 & 1.27 \\
\hline & 1 & 10.98 & 9.73 & 0.72 & 9.73 & 8.83 & 0.74 & 9.73 & 8.47 & 1.13 & 9.73 & 8.39 & 1.35 \\
\hline & 2 & 9.06 & 8.40 & 0.62 & 8.87 & 8.01 & 0.68 & 8.87 & 7.62 & 0.99 & 8.87 & 7.36 & 1.55 \\
\hline & 3 & 9.40 & 7.80 & 0.71 & 9.35 & 7.48 & 0.90 & 9.35 & 7.20 & 1.12 & 9.35 & 7.11 & 1.34 \\
\hline & 4 & 11.96 & 9.28 & 0.71 & 10.05 & 8.72 & 0.94 & 9.00 & 7.92 & 0.95 & 8.96 & 7.43 & 1.34 \\
\hline \multirow[t]{5}{*}{40} & 0 & 10.27 & 9.44 & 0.26 & 9.60 & 8.80 & 0.78 & 9.60 & 8.54 & 0.93 & 9.60 & 8.46 & 1.15 \\
\hline & 1 & 10.51 & 9.67 & 0.67 & 9.73 & 9.07 & 0.56 & 9.73 & 8.80 & 0.98 & 9.73 & 8.76 & 1.09 \\
\hline & 2 & 9.49 & 9.10 & 0.65 & 9.05 & 8.53 & 0.62 & 8.98 & 8.11 & 0.88 & 8.98 & 7.88 & 1.42 \\
\hline & 3 & 9.35 & 8.14 & 0.67 & 9.35 & 7.95 & 0.73 & 9.35 & 7.79 & 1.00 & 9.35 & 7.76 & 1.12 \\
\hline & 4 & 10.59 & 9.16 & 0.92 & 9.92 & 8.59 & 0.89 & 8.96 & 7.91 & 0.81 & 8.96 & 7.64 & 1.07 \\
\hline
\end{tabular}

